\title{
Spät- und Postglazial im Geiseltal (mittleres Elbe-Saalegebiet)
}

\author{
Dietrich Mania, Maria Seifert, Matthias ThomaE*) \\ Pleistocene, Weichselian, Pleniglacial, Late Glacial, Holocene, Stratigraphy, vegetation sequence, \\ mollusc fauna, habitat. \\ Middle Elbe-Saale regio
}

Kurzfassung: Im westlichen Geiseltal (mittleres ElbeSaalegebiet, westlich von Merseburg) waren spätglaziale und holozäne Serien großflächig aufgeschlossen. Sie lagen auf der Niederterrasse und dem Löß der Weichselkaltzeit. Im wesentlichen handelte es sich um limnische und telmatische Sedimente. Sie zeigten eine Gliederung in fünf Sedimentzyklen mit zwei präböllingzeitlichen Intervallen (Müchelner Intervalle 1 und 2), mit Bölling, Alleröd und Holozän. Die Kaltphasen waren durch Beckenschluffe, die warmen Phasen durch Mudden und Torfe, seit dem Alleröd auch durch Charatravertinsande gekennzeichnet. Im Alleröd war die Laacher-See-Tephra eingelagert. In der Jüngeren Dryas-Zeit entstanden zum letzten Male Frostspaltennetze. Für Ältere Dryas-Zeit, Alleröd und Jüngere Dryas-Zeit wurde mit Hilfe der Pollenanalyse die Vegetationsgeschichte ermittelt. Alle Horizonte sind durch Molluskenfaunen dokumentiert, die in ihrer wechselnden $\mathrm{Zu}$ sammensetzung den detaillierten Klimaablauf erkennen lassen. Zugleich liegt eine der bedeutendsten spätglazialen Molluskensukzessionen Mitteleuropas vor.

\section{[Late Glacial and Holocene in the Geisel Valley, Middle Elbe-Saale Region]}

Abstract: In the western part of the valley of the Geisel (area of the Saale river, west of Merseburg) late glacial and holocene series were exposed over large areas. They lay on the lower terrace and on the loess originating from the Weichselian. They were built up in cycles displaying a division into five cycles with two intervalls from the Pre-Bölling (Mücheln Intervalls 1 and 2), Bölling, Alleröd and Holocene. The glacial phases were characterized by basin silt, the warm phases exhibited gyttjas and peat with chara-travertin since Alleröd. During Alleröd, the tuff depositions from the Laacher See eruption occured. In the Younger Dryas, a net of frost cracks formed for the last time. Pollen analyses established the vegetation sequence valid for Early Dryas, Alleröd und Younger Dryas. All horizons are documented by mollusc fauna revealing the detailed climatic course through their changing composition. In addition, they represent one of the most important late glacial mollusc successions of Central Europe.

*) Anschriften der Verfasser: Dr. rer. nat. D. ManIA, Ibrahimstr. 29, 09599 Jena. Geol. Ing. M. SEIFERT, Sächsisches Landesamt für Umwelt und Geologie, Halsbrücker Str. 31 a, D - 09599 Freiberg/Sa. Dr. rer. nat. M. Thomae, Straße der Jugend 16, D - 0-4401 Vockerode.
Inhalt

$1 \quad$ Einleitung

2 Lagerungsverhältnisse (ThomaE. M.\&MANIA, D.)

3 Die Sedimentabfolge (Mania, D. \& Thomae. M)

3.1 Die Flußschotter

3.2 Das Spätglazial

3.2.1 Mücheln 1

3.2.2 Mücheln 2

3.2.3 Mücheln 3

3.2.4 Mücheln 4

3.2.5 Mücheln 5

3.3 Parallelbefunde

4 Vegetationsgeschichte (SEIFERT, M.)

4.1 - Die pollenanalytischen Abschnitte

4.1.1 Salix-Phase

3.1.2 Betula-Phase

4.1.3 Pinus-Phase

4.1.4 Übergangsphase

4.1.5 Eichenmischwaldphase

4.2 Zur Vegetationsgeschichte

5 Die Molluskenfauna und ihre Lebensräume (MANiA, D.)

5.1 Hochglazial

5.2 Müchelner Intervall 1 (Profil A)

5.3 Kaltphase zwischen den Müchelner Intervallen 1 und 2

5.4 Müchelner Intervall 2 (Profil A und B)

5.5 Älteste Dryas-Zeit

5.6 Bölling-Interstadial

5.7 Ältere Dryas-Zeit

5.8 Alleröd-Interstadial

5.9 Jüngere Dryas-Zeit

5.10 Holozän

6 Schriftenverzeichnis

\section{Einleitung}

Das Tertiärbecken des Geiseltals befindet sich nur wenige Kilometer von der Leipziger Tieflandbucht entfernt am Nordostrand des Hügellandes, das der Mittelgebirgszone vorgelagert ist. Das Becken ist 


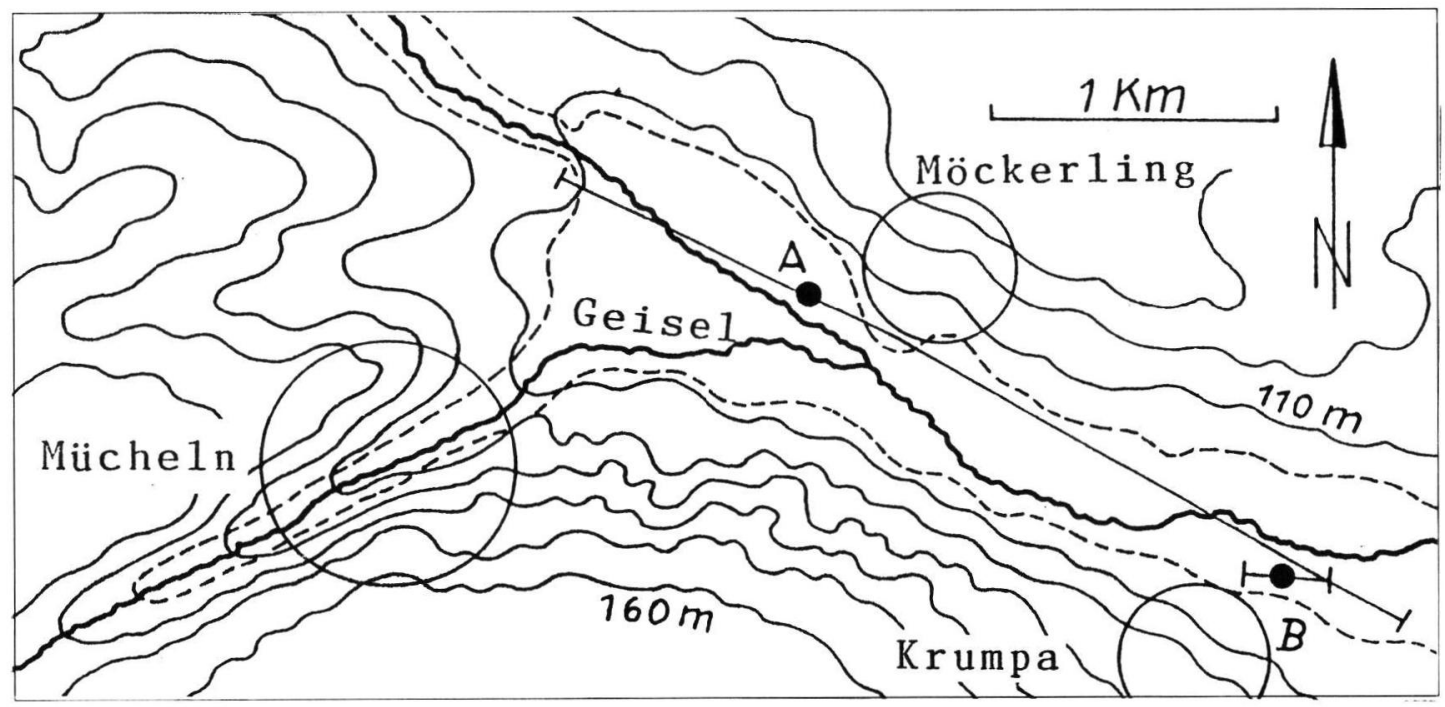

Abb. 1: Das westliche Geiseltal. Lage des Schnittes durch die Geiselaue (nach Tagebauaufschlüssen von 1965 - 1967 und 1986-1992). A und B: Profile A und B.

zwischen die Querfurt-Freyburger Muschelkalkmulde und die Merseburger Buntsandsteinplatte eingesenkt (s. Abb. 1).

Die Geisel, ein kleines Flüßchen von etwa $20 \mathrm{~km}$ Länge, entspringt am Rand der Muschelkalkstufe. Sie folgte im wesentlichen der Beckenachse in östlicher, dann nordöstlicher Richtung und floß der Saale durch eine sumpfige, mit Riedflächen und Auwäldern durchsetzte Niederung zu. Heute ist diese Landschaft durch den Braunkohlenbergbau devastiert und weitgehend verändert.

In den Tagebauen waren die quartären Deckschichten viele Jahrzehnte hindurch aufgeschlossen. Dadurch wurden in den sechziger Jahren im westlichen Teil des Geiseltals, im Tagebau Mücheln, jungquartäre Ablagerungen über ein Strecke von mehr als $3 \mathrm{~km}$ Länge zugänglich. Sie bestanden vorwiegend aus Schottern der Geisel sowie spätglazialen und holozänen Beckenablagerungen. Ihr Fossilreichtum veranlaßte ihre detaillierte Untersuchung (MANIA \& ToEPfEr 1971, Mania 1973). Seit 1986 wurde dieser Aufschluß in östlicher Richtung um einige Kilometer erweitert. In den spätglazialen Ablagerungen konnte der vulkanische Tuff vom Laacher See (Eifel) nachgewiesen werden. Bereits H. MÜLLER (1953) beobachtete diesen Tuff bei Frankleben im Geiseltal, als er dort mit vegetationsgeschichtlichen Untersuchungen des mitteldeutschen Trockengebiets begann.

Mit Hilfe des Tuffs konnten die spätglazialen Ablagerungen unter dem Holozän genauer stratigraphisch gegliedert werden. Es stellten sich flache, bis über 100 m lange Becken mit allerödzeitlichen Sedi- mentabfolgen heraus. In tieferen Beckenteilen wurden die Ablagerungen älterer Wärmeschwankungen gefunden, die dem Alleröd unmittelbar vorausgingen. Einschließlich Alleröd wurden vier spätglaziale Sedimentationsfolgen festgestellt. Sie waren zyklisch aufgebaut und begannen jeweils über einer Denudationsfläche mit geringmächtigen Sanden und Beckenschluffen, enthielten im mittleren Teil organogene limnische und telmatische Ablagerungen (Schluff-, Ton-, Kalkmudden, Charakalke, Grobdetritusmudden, Torfe, Anmoore) und endeten wieder mit Beckenschluffen. Die mittleren Abschnitte stellen die Wärmeschwankungen dar, die begrenzenden Abschnitte die Kaltphasen. Gelegentlich wurden auch Froststrukturen beobachtet.

\section{Lagerungsverhältnisse (Thomae, M., \& Mania, D.)}

Die jungquartäre Abfolge erstreckte sich vom westlichen Rand der Aue bei Mücheln in östlicher Richtung längs durch das Tal bis in die Gegend von Krumpa (s. Abb. 2). Sie wurde etwa 15 m mächtig und gliederte sich in drei Einheiten, die flächendeckend verbreitet waren (s. Abb. 3 und 4):

1. eine untere Decke aus sandigen Flußschottern der Geisel (2 - $6 \mathrm{~m})$,

2. eine mittlere Decke aus Beckenablagerungen des Spätglazials (2 - $8 \mathrm{~m}$ ),

3. eine obere Decke aus verschiedenen limnischtelmatisch-fluviatilen Sedimenten des Holozäns $(4-8 m)$. 
Im westlichen Bereich lagerte die Serie direkt auf dem Tertiär, das hier aus Kohle bestand. In östlicher Richtung wurde das Jungquartär mehr und mehr von älteren quartären Ablagerungen, so der saalezeitlichen Grundmoräne, unterlagert.

An den Talrändern schob sich zwischen die Flußschotter und das Spätglazial die jungpleistozäne Lößdecke ein. Sie erreicht in der Umgebung des Geiseltals erhebliche Mächtigkeiten ( 2 - $6 \mathrm{~m}$ ).

Während die holozäne Decke im allgemeinen flach und wenig gestört gelagert war sowie nur geringe Mächtigkeitsschwankungen aufwies, waren die unteren Decken in ihrer Lagerung gestört. Sie wurden durch Braunkohlenrücken aufgewölbt oder sogar unterbrochen. Diese Lagerungsstörungen sind auf Mollisoldiapirismus (Kohlediapirismus, Karbokinese) zurückzuführen (EISMANN 1978, RuchHOlz 1977, Thomae 1986, 1990). Die Randsenken im umlaufenden Streichen der aufgestiegenen Beulen und Rücken waren im westlichen Geiseltal mit spätglazialen Sedimenten gefüllt. An den tiefsten Stellen erreichten diese Beckenfüllungen Mächtigkeiten von $8 \mathrm{~m}$ zugleich war hier die Möglichkeit zur zeitlichen Dehnung des Sedimentationsgeschehens gegeben. So konnten sich kurzfristige, klimatisch gesteuerte
Ereignisse in speziellen Sedimentzyklen niederschlagen. Die holozäne Sedimentation begann ebenfalls in den Randsenken. Nach und nach klang aber die Karbokinese aus und eine flächendeckende Sedimentation überwog. Nur im westlichen Bereich (vgl. Abb. 3) ließ sich anhaltender Kohleaufstieg und damit verbundene Senkenbildung bis zum 16. Jahrhundert nachweisen. Erst ab dieser Zeit griff der obere Auelehm, der an seiner Basis Scherben des 16. bis 18. Jahrhunderts enthielt, ungestört über die Strukturen hinweg.

Der Kohlediapirismus war an das Durchfrieren der Kohle und das anschließende Auftauen des Bodeneises während der jeweiligen spätglazialen und frühwarmzeitlichen Phasen geknüpft. Dabei traten die gravitativen Prozesse in der destrukturierten und wassergesättigten Kohle unter Sedimentbelastung ein.

\section{Die Sedimentabfolge (Mania, D., \& Thomae, M.)}

\subsection{Die Flußschotter}

Die gröberen Fraktionen der Schotter bestanden zu $75 \%$ aus Muschelkalk, der Rest sowie die feineren

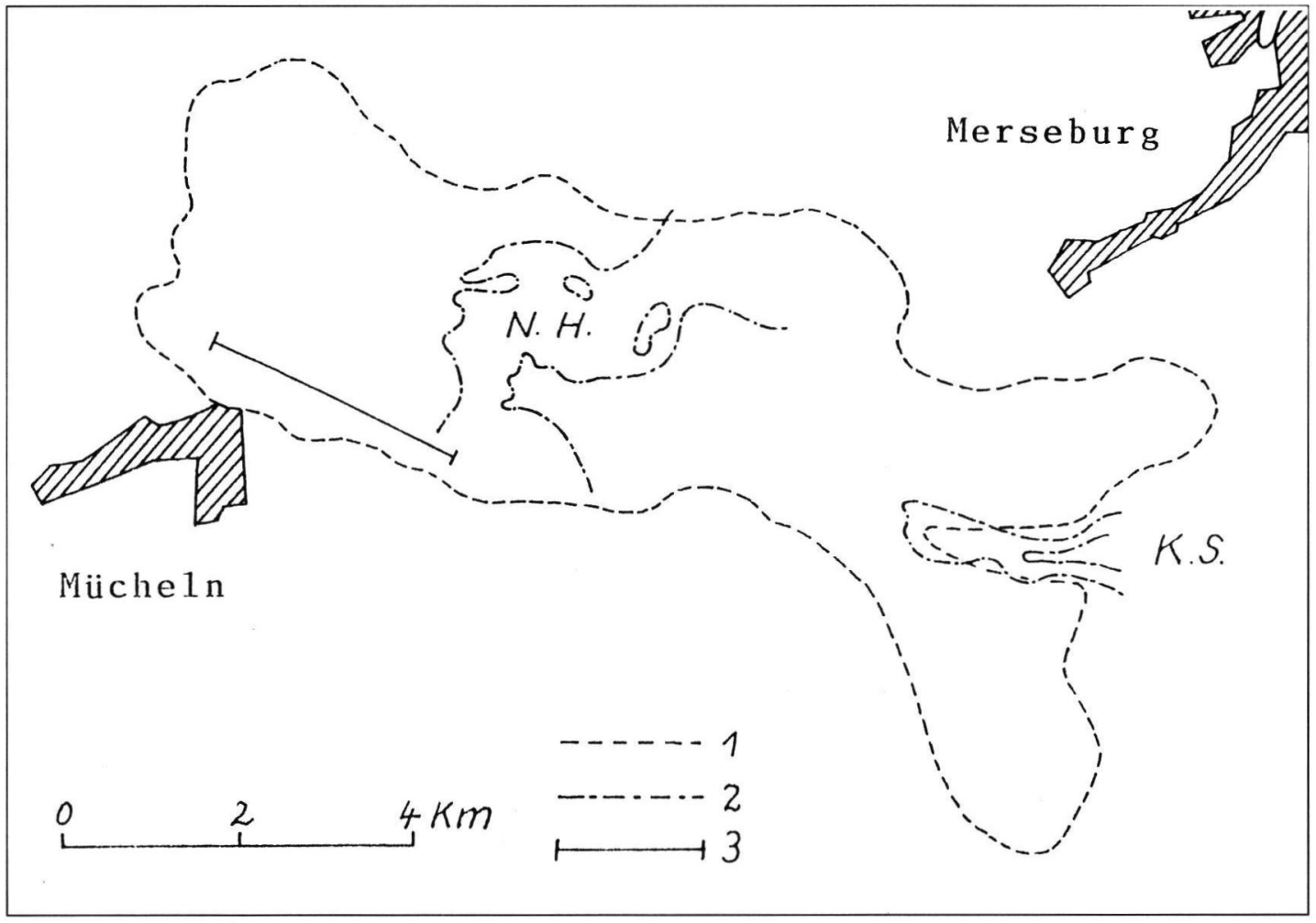

Abb. 2: Lage des Schnittes im Geiseltalbecken. 1 2-m-Isopache der Kohle, 2 Schwellengebiete (N. H. Neumark-Hauptschwelle, K. S. Kaynaer Schwelle), 3 Profillinie in den spät- und nacheiszeitlichen Ablagerungen des westlichen Geiseltals. 


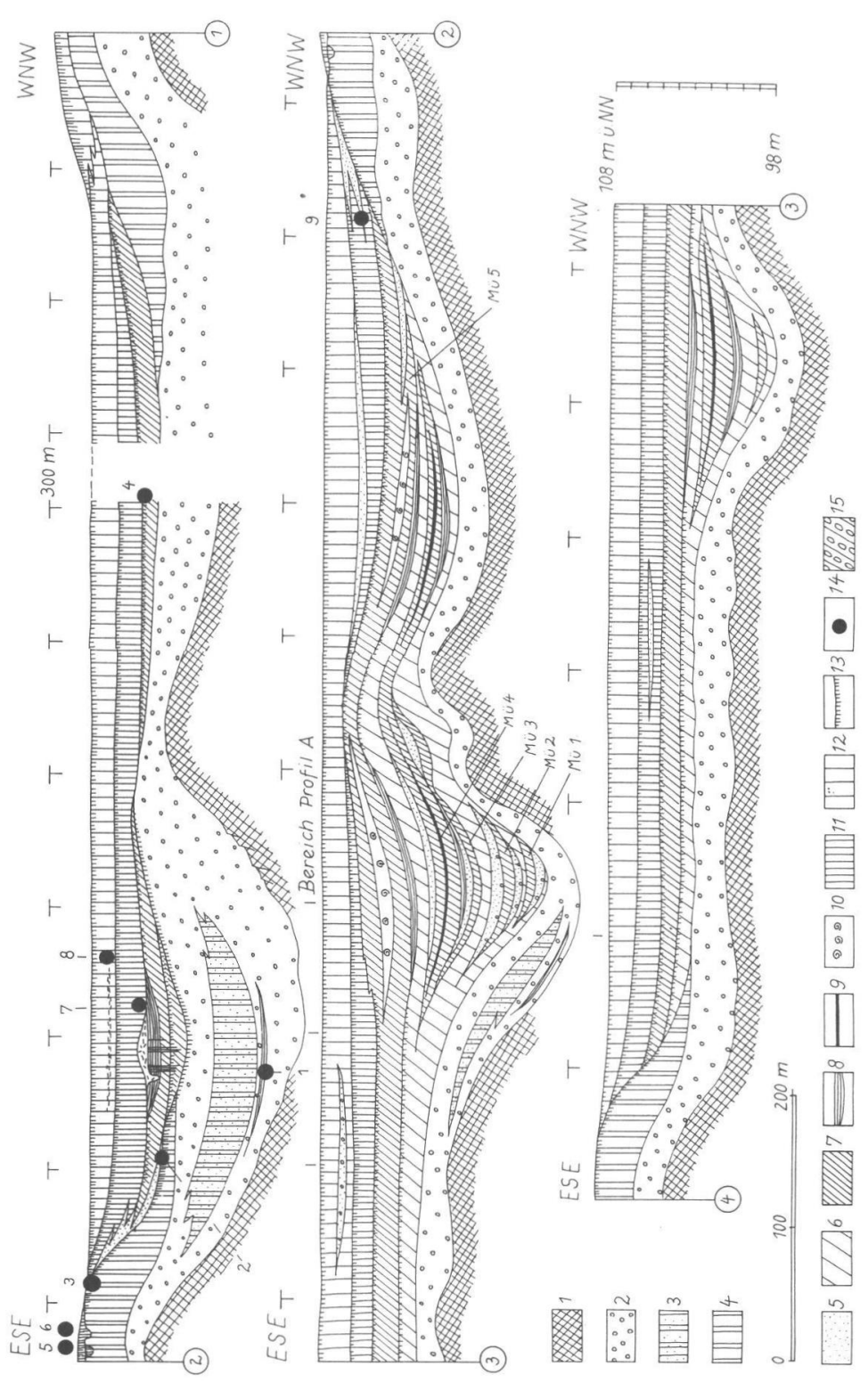

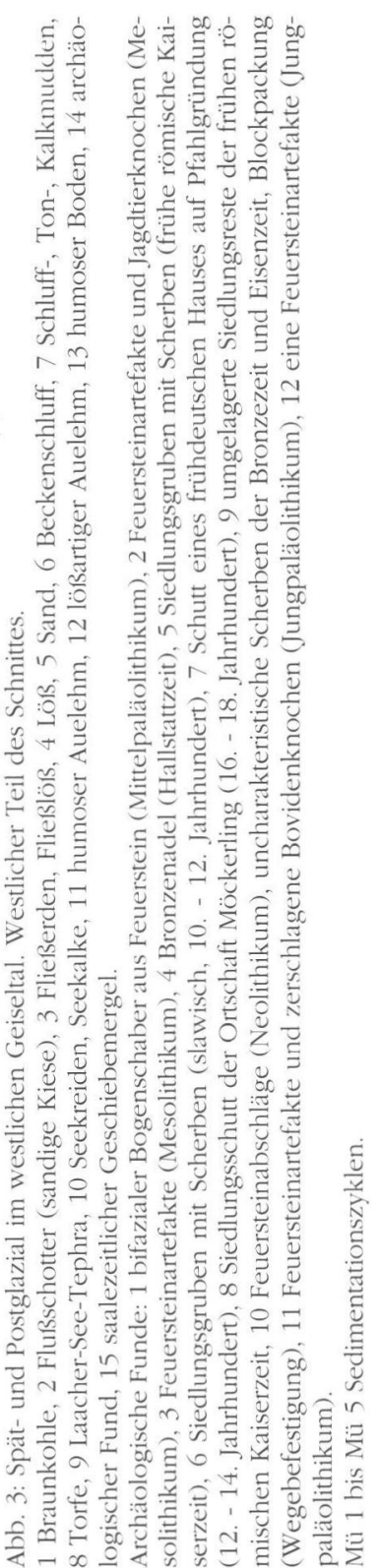

Fraktionen aus aufgearbeiteten pleistozänen und tertiären Sedimenten (überwiegend Quarz, ferner Feuerstein, Kristallin, Quarzit u. a.). Die Schotter entsprechen der weichselzeitlichen Niederterrasse der Flüsse im Saalegebiet. Sie entstanden unter den besonderen Bedingungen des Frühglazials, dem miteinander gekoppelten Ablauf von Erosion, Transport und Akkumulation. Im unteren Teil der Geiselniederterrasse wurde bei Mücheln (Profil A) ein Torf-Grobdetritusmudde-Horizont beobachtet, der Kiefer- und Birkenhölzer enthielt. Er ist mit einem frühweichselzeitlichen Interstadial der Zeit vor 50000 B. P. gleichzusetzen. In die gleiche Zeit gehört ein mittelpaläolithischer bifazialer Bogenschaber aus Feuerstein, der im Schotter gefunden wurde (MANiA 1968). Im oberen Teil der Schotter waren sandig-kiesige, lehmige Fließerden eingebettet. Hier traten auch Froststrukturen auf, wie Kryoturbationen und Frostspalten.

\subsection{Das Spätglazial}

Die spätglazialen Beckenbildungen konnten nicht nur mit Hilfe des Laacher-See-Tuffes datiert werden, sondern auch dadurch, daß sie im Talrandbereich 


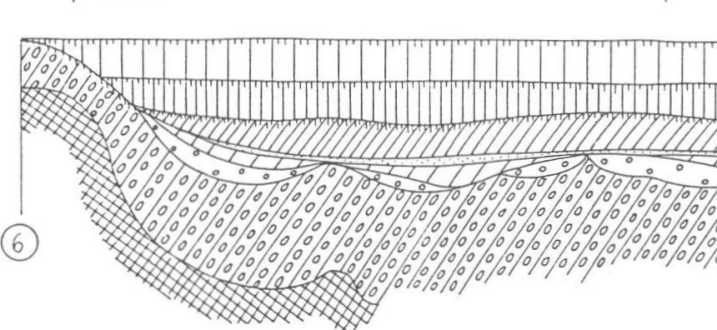

(6)

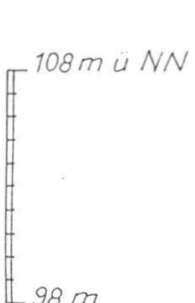

$100 \mathrm{~m}$<smiles>C1=CCC[CH-]1</smiles>

Bereich Profil B

10

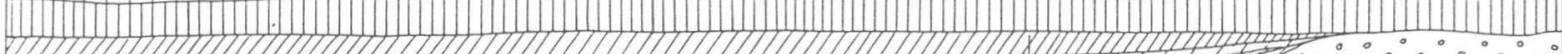
4 (3)

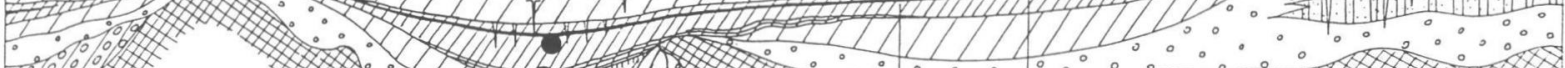

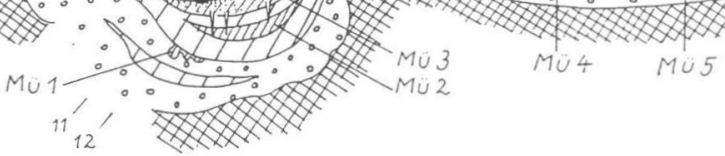


diskordant den hochweichselzeitlichen Löß überlagerten (s. Abb. 3 - 5).

\subsubsection{Mücheln 1}

Der unterste Zyklus der spätglazialen Abfolge begann und endete mit Schluffen, aus denen sich im mittleren Teil eine bis $40 \mathrm{~cm}$ mächtige tonige Schluffmudde entwickelte. Sie enthielt größere Mengen an pflanzlichem Grobdetritus, wie Gräser, Moose und Holzreste von Sträuchern, besonders strauchförmigen Weiden und Birken (Profil A). Im Profil B war dieser Horizont nur in Form kryoturbat veränderter Muddenreste unter dem Basalschluff vorhanden.

Bei den Beckenschluffen, auch der höher im Profil folgenden Horizonte, handelt es sich offenbar um umgelagerten, teilweise aber auch eingewehten Löß.

\subsubsection{Mücheln 2}

Über sandigen Einschwemmungen entstanden wieder Beckenschluffe, aus diesen ging eine $50 \mathrm{~cm}$ mächtige sandige Grobdetritusmudde hervor. Sie enthielt zahlreiche Holzreste von Polarsträuchern. Am Profil B war der Horizont als tonige grobdetritusreiche Schluffmudde ausgebildet. Sie ging nach oben in einen schluffigen Muddesand mit Holzdetritus von Sträuchern und eingelagerten tonigen Schlufflinsen über. Das deutet auf eine sumpfige Fazies, die sich aus dem flachen Wasserbecken entwickelte. Danach wurden Feinsande eingeschwemmt, und Froststrukturen entstanden, so Kryoturbationen und einige bis $1,5 \mathrm{~m}$ tiefe Frostspalten.

\subsubsection{Mücheln 3}

Dieser Horizont war im westlichen Teil des Aufschlusses als deutlicher Zyklus ausgebildet, dessen organogene Sedimente von Beckenschluffen unterund überlagert waren. Mit diesen erreichte er Mächtigkeiten von 1,5 bis $2 \mathrm{~m}$. Charakteristisch waren tonige Schluffmudden mit bereits recht hohem Anteil an organogenem Material, Grobdetritusmudden, Moos- und Grastorfe, die wiederholt Holzreste, auch von Baumbirken, enthielten. Mitunter waren die Mudden mit Travertinaggregaten durchsetzt, die autochthon im Wasser ausgeschieden worden waren. Dadurch erhielten die Mudden eine sandige Beschaffenheit, der Zyklus war zum darüber liegenden Horizont auch durch Sande und kiesige Sande getrennt.

Im östlichen Teil des Aufschlusses, bei Krumpa, war der Mücheln-3-Zyklus weniger deutlich ausgebildet.
Meist verbarg er sich in einem relativ mächtigen tiefschwarzen Anmoor mit hellgrauer Bleichzone im Unterboden und Torflinsen im Humushorizont. Dieser Boden hielt über weite Strecken aus und lag an der Basis des folgenden Zyklus (vgl. Abb. 4). Im Bereich des Profils B war der Boden weniger intensiv entwickelt; unter ihm kamen fossilführende Muddesande des Zyklus 3 zum Vorschein.

\subsubsection{Mücheln 4}

Als jüngster und am weitesten entwickelter spätglazialer Zyklus hatte er auch die größte Verbreitung in den Randsenken. Er begann großflächig mit 30 bis $50 \mathrm{~cm}$ mächtigen sandigen Torfen, die Gräser, Moose und Strauchreste enthielten. Außerdem kamen einige uncharakteristische Feuersteinartefakte und zerschlagene Boviden-Knochen zum Vorschein.

Direkt über dem Torf folgten Grobdetritusmudden mit dünnen Moos- (z. B. Braunmoos-) und "Schilf"torflagen sowie zahlreichen Holzresten von Weiden und Baumbirken. Aus ihnen entwickelten sich bis $2 \mathrm{~m}$ mächtige Mudden, zunächst als Wechselfolge von dünnen graubraunen Feindetritusmudden und etwas mächtigeren gelblich-braunen oder weißlichen Kalkmudden. Nach oben nahmen die Feindetritusmudden immer mehr zugunsten gelber Charamudden und Charakalke ab, in denen sich auch sandig-kiesige Travertinaggregate befanden. Im oberen Teil der Charakalke lagerte die Tephra aus dem Laacher See. Sie bildete eine zusammenhängende Schicht von 5 bis $10 \mathrm{~cm}$ Mächtigkeit. Über ihr folgten nochmals 20 bis $30 \mathrm{~cm}$ mächtige Charasande und Kalkmudden, dann wieder braune tonige Feindetritus- und Schluffmudden. In der gesamten Folge kamen Holzreste von Birken, weniger häufig von Kiefern vor. Im Randbereich der Becken gingen die Kalkmudden in fossilreiche Grobdetritusmudden über, die in pflanzenreichen Sümpfen abgelagert wurden und gelegentlich Horizonte aus einem Ast- und Stammgewirr von Birken, Kiefern und Weiden bedeckten. Diese deuten auf bruchwaldartige Gehölze in der sumpfigen Umgebung der Becken hin. Auch Flachmoortorfe kamen vor. In den Charakalken wurden einige Feuersteinklingen des Jungpaläolithikums gefunden.

In der Regel wurden die Mudden von etwa 0,3 bis 1,0 m mächtigen Beckenschluffen bedeckt. Von ihrer Basis gingen in den Becken starke Kryoturbationen und bis $1 \mathrm{~m}$ tiefe Spaltennetze aus. Die Ränder der Spalten weisen Aufpressungen auf. Es liegen eindeutig Bodeneiswirkungen vor (s. Abb. 6).

Im Bereich des Profils B war eine weitere feinstratigraphische Gliederung dieses Abschnitts zu beobachten: Auf den Mudden der vorangegangenen Wär- 


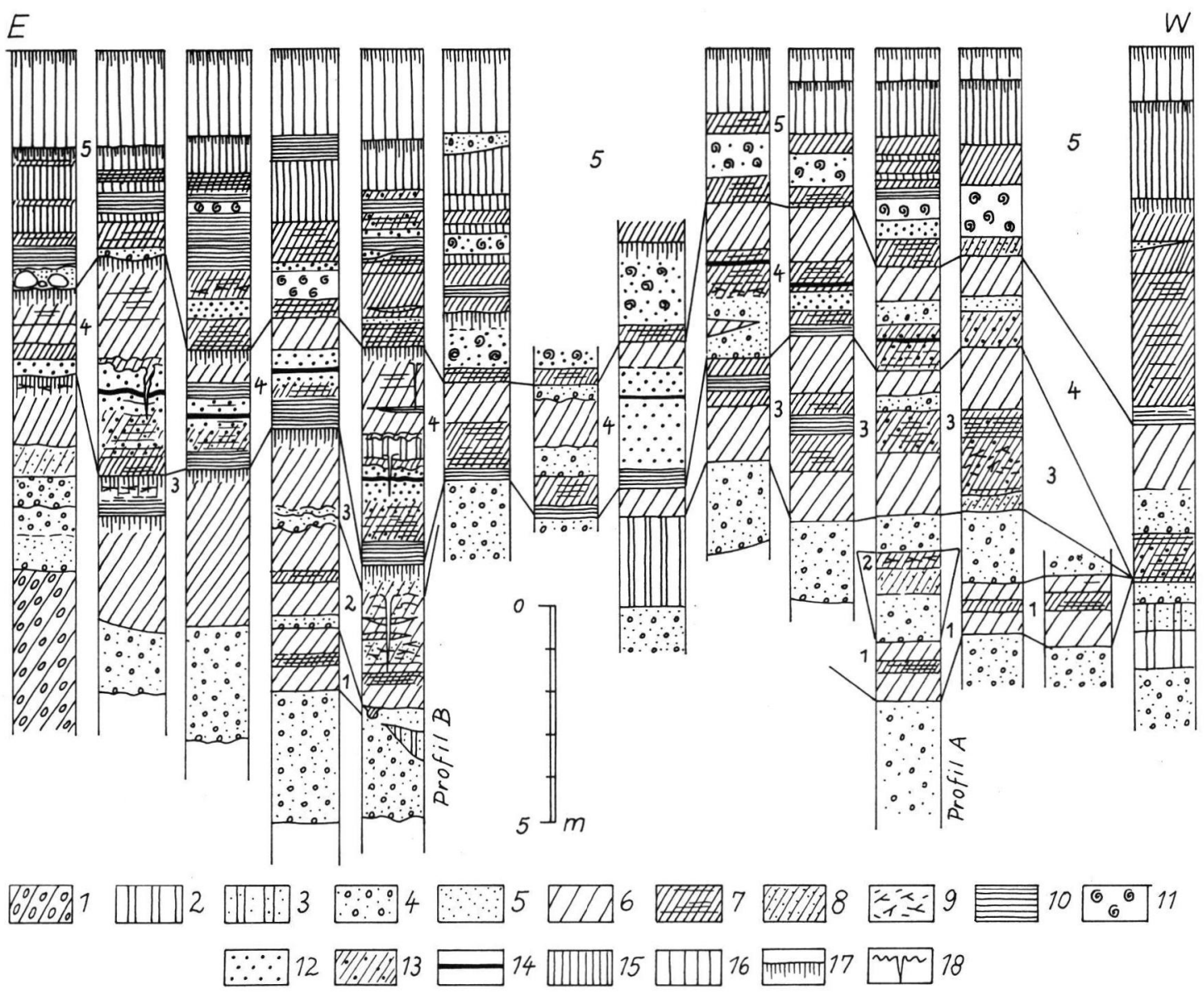

Abb. 5: Einige spezielle Profile aus dem Spät- und Postglazial des westlichen Geiseltals.

1 saalezeitlicher Geschiebemergel, 2 Löß, 3 Fließlöß, Fließerde, 4 sandige Kiese, 5 Sande, 6 Beckenschluff, 7 Schluff-, Tonund Kalkmudden, z. T. Grobdetritusmudden, 8 Muddesande, 9 Grobdetritus aus Hölzern, 10 Torfe, 11 Seekalk, 12 Travertinsand, Charasand, 13 Mudden mit Travertinaggregaten, 14 Laacher-See-Tephra, 15 unterer humoser Auelehm, 16 oberer lößartiger Auelehm, 17 humoser Boden, 18 Froststrukturen, Frostspalten.

Zahlen an den Profilen: Sedimentationszyklen Mücheln 1 - 5

meschwankung lag ein $40 \mathrm{~cm}$ mächtiger reiner Schluff, der als ein Löß angesprochen werden kann. An seiner Basis befand sich ein Frostspaltennetz. Über die Spaltenöffnungen griffen $5 \mathrm{~cm}$ mächtige, millimeterdünn gewarvte verschwemmte Feinsande und Schluffe hinweg. Etwa 20 Warven konnten gezählt werden. Diese Erscheinung war über größere Strecken hin zu beobachten und ist offenbar als ein Ergebnis zirkulierender Oberflächenwässer des sommerlichen Auftaubodens anzusehen. Über ihr folgte der Hauptteil des ungeschichteten Lößes. Er wurde von einer zweiten gewarvten, kryoturbat veränderten Schluff-Feinsandlage abgeschlossen. Diskordant darüber folgten $2 \mathrm{~m}$ mächtige kalkhaltige Schluffmudden, zunächst ungeschichtet und minerogen, nach oben aber mit undeutlicher Schichtung und Zunahme des organogenen Sedimentanteils, besonders von eingelagertem Grobdetritus, in dem Blätter von Dryas octopetala und Zwergsträuchern (Birken, Weiden) überwogen. Auch dünne Moostorf- und Grastorflagen waren eingeschaltet. Etwa im Mittelteil ließ die Schichtung wieder nach, und mehr und mehr entwickelte sich eine tonige Schluffmudde. Oben wurde sie von einem tiefgründig gebleichten Anmoor-Humusgley mit schwarzem AHorizont und Baumwurzeln abgeschlossen. Dicht unter dem Boden wurde nochmals eine schmale Frostspalte beobachtet.

Der Boden selbst stammt aus dem Früh- und Mittelholozän. Auf ihm wurden frühgeschichtliche Scherben gefunden und eine aus großen Findlings- 
blöcken zusammengepackte Blocklage, offenbar eine Wegbefestigung im Sumpf.

\subsubsection{Mücheln 5 (Holozän)}

Die holozäne Serie ist sehr vielgestaltig aufgebaut. In den tiefsten Beckenbereichen begann sie mit Flachmoortorf und einer den spätglazialen Ablagerungen sehr ähnlichen Grobdetritusmudde (Präboreal) Aus ihr gingen schluffige bis tonige Sumpfund Seekalke, Travertinsande sowie Grobdetritusmudden und Torflagen hervor (Boreal). Der Horizont lief uferwärts in einen schwarzen Anmoorboden aus, welcher bei Möckerling mesolithische Funde enthielt: Feuersteinartefakte und zerschlagene Knochen von Wildschwein, Rothirsch, Reh, Auerochs und Wildpferd (MANIA \& TOEPFER 1971). Schilftorfe, von See- und Sumpfkalken unterbrochen, gehören ins Atlantikum. Nun folgte wieder ein schwarzer Aueboden, aus dem bronzezeitliche (hallstattzeitliche) Funde stammen (Subboreal). Darüber lagerten auelehmartige graue Schluffe, Schilftorfe und Grobdetritusmudden. Ein Teil dieser Ablagerungen gehört bereits in das Subatlantikum, denn er verzahnt sich mit sandig-kiesigen Abspülhorizonten, in denen Scherben der römischen Kaiserzeit gefunden wurden. Über der Folge setzten schließlich zwei mächtige Auelehme ein, ein unterer, durch abgetragenes Bodenmaterial grau gefärbter und ein oberer lößartiger, gelbbrauner Auelehm (Subrezent). Der untere griff über einen Pfahlrost mit Schutt eines Aufbaues und Scherben des 12./13. Jahrhunderts hinweg; in der Basis des oberen lagerten Schuttschichten des 16. bis 18. Jahrhunderts. In der gesamten holozänen Folge traten hin und wieder linsenförmige, geringmächtige Sand- und Schotterkörper der Geisel auf.

\subsection{Parallelbefunde}

Spätglaziale Serien wurden aus dem Ascherslebener See (Mania 1967), aus den Nebentälern der mittleren Saale (Mania \& Stechemesser 1969, 1970), aus den Bergländern der Hainleite, vom Unterharz (MANIA 1972 , 1973) und neuerdings aus der Mißaue-Senke am Elm (Thieme, H., mdl. Mitt.) bekannt.

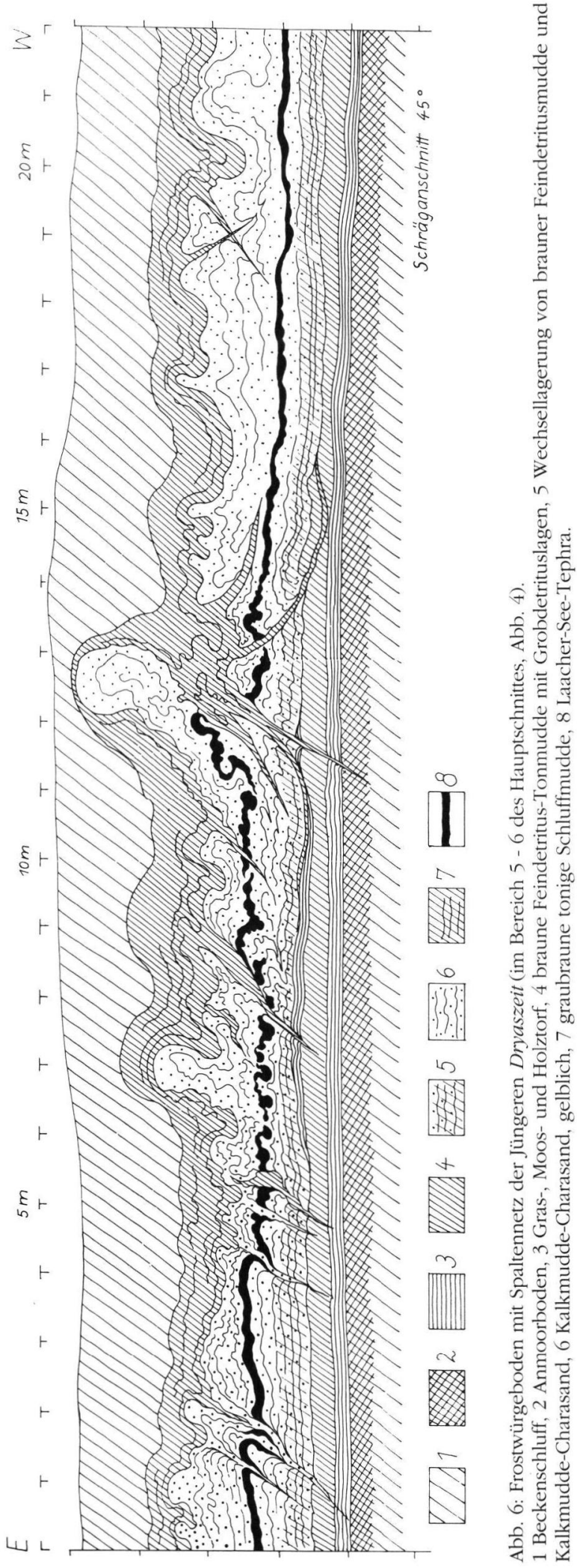


Den Abfolgen aus dem Geiseltal sind die spätglazialen Ablagerungen des Ascherslebener Sees sehr ähnlich. Sie stammen auch aus einem sehr ähnlichen Bildungsraum. Auch hier ist die obere der vorhandenen zwei Folgen durch die eingelagerte Laacher Tephra datierbar (MülLER 1953, FRECHEN 1953, MANIA 1967), sind bereits Kalkmudden und Charakalke entwickelt und die kaltzeitlichen Phasen zwischen den Wärmeschwankungen durch Beckenschluffe und Schluffmudden, auch sandige bis kiesige Horizonte und Denudationsflächen gekennzeichnet. Im Schluffhorizont der Jüngeren Dryas-Zeit war ein Spaltennetz durch Bodenfrosteinwirkung ausgebildet. Die beiden Wärmeschwankungen von Plinz südlich Jena und vom Eskaborner Berg (Unterharz) enthielten ebenfalls geringmächtige Lagen von Travertinsanden.

Wie im Ascherslebener See ist im Geiseltal der oberste spätglaziale Zyklus (Mücheln 4) dem Alleröd zuzuweisen. Im allgemeinen gehört der darunter befindliche der Böllingschwankung an (Mücheln 3). Die beiden Schwankungen unter dieser klassischen Abfolge des Spätglazials wurden im Saalegebiet bisher nicht nachgewiesen. Sie vermitteln zwischen dem Hochglazial und dem Spätglazial und wurden auf Grund ihrer schwachen Entwicklung als "Müchelner Intervalle 1 und 2" bezeichnet. Sie müssen in die Zeit kurz vor 13500 B. P. gehören.

\section{Vegetationsgeschichte (SEIFERT, M.)}

Zur pollenanalytischen Untersuchung ${ }^{*}$ ) wurde das Profil B ausgewählt. Wie schon MülLer (1953) bei seinen Proben von Frankleben aus dem Geiseltal feststellen konnte, enthielten zahlreiche Abschnitte der beprobten Abfolge Fremdpollen aus dem Tertiär (Eozän), die aus den benachbarten Kohlediapiren stammen. Das betraf die Proben 1 bis 27 (s. Abb. 7) (Mücheln 1 bis 3), die keine Pollenanalyse ermöglichten. Im oberen Profilteil (Proben 31 bis 75, Mücheln 4 und 5) waren Pollendichte und Erhaltungszustand der quartären Pollen relativ gut.

\subsection{Die pollenanalytischen Abschnitte}

\subsubsection{Salix-Phase}

Die Folge beginnt im zersetzten Basistorf mit einem Salix-Maximum. Pinus und Betula weisen noch niedrige Werte auf. Hippophaë, Populus, Juniperus und Betula nana (?) kommen vor. Der sehr hohe Kräuteranteil zu Beginn des Abschnitts besteht aus Gramineen, Pollen des Carex-Typs, Artemisia, Heli-

\footnotetext{
*) Mitteilung der Geologischen Landesuntersuchung, Freiberg/Sa., Nr. 773/92. - Die Proben wurden im Sächs. Landesamt f. Umwelt u. Geologie, Freiberg/Sa. aufbereitet. Behandlung mit HNO3 zur Mazerierung der Pollen und Entfernung des Kalkgehalts, danach mit HF und HC1 zur Lösung von Silikaten und Kieselsäure; Huminsäuren wurden durch das Erhitzen von Kalilauge extrahiert. Aus finanziellen Gründen konnte nur jede zwcitc Probe untersucht werden.
}

anthemum, Filipendula, Umbelliferae, Thalictrum und Tubuliflorae.

\subsubsection{Betula-Phase}

Über dem Torf steigen die Betula-Werte rasch an. Die hohen Salix-Werte sinken ab. Auch die Kräuter gehen etwas zurück. Pinus beginnt sich stärker auszubreiten. Auffällig sind noch Betula nana (?)-Pollen. Nach ihrem Maximum von bis $60 \%$ weicht $B e$ tula zurück, während die Pinus-Kurve weiter ansteigt.

\subsubsection{Pinus-Phase}

Im Bereich der Charakalke dominiert Pinus und erreicht Maximalwerte, die jeweils mit kleineren Birken-Gipfeln zusammenfallen. Populus (wahrscheinlich $P$. tremula) ist nachweisbar. Salix geht weiter zurück. Der Anteil der Kräuter/Gräser bleibt hoch und steigt nach oben stetig an. Die Gramineen erreichen im Bereich des Laacher Tuffs einen Gipfelwert von über $60 \%$, der alle anderen Pollenkurven in der Relativierung unterdrückt. Pinus hätte sonst ohne diese Überprägung viel höhere Werte. So werden statt dessen nachfolgend wieder leicht ansteigende Kurven von Betula und Pinus hervorgerufen, obwohl beide jetzt allgemein im Rückgang begriffen sind. Populus ist noch vorhanden.

Mit Beginn des ersten Pinus-Anstiegs und der Betula-Ausbreitung treten Sphagnum, Equisetum, Typha latifolia und Myriophyllum auf. Später werden Sparganium und Ceratophyllum häufiger. Im Gewässer breiteten sich dichte Chara-Rasen aus. Polypodiaceen sind durchgehend vorhanden, ebenso Süßwasserplankter mit gelegentlich sehr hohen Werten.

\subsection{4 Übergangsphase}

Der nach oben anschließende Bereich (Schluff-Folge) ist zunächst durch ein Minimum von Betula und stark absinkenden Pinus-Werten gekennzeichnet. Gleichzeitig kommen Salix- und Kräuterpollen wieder reichlicher vor, so von Artemisia, Chenopodiaceae, Thalictrum, Filipendula, Potentilla u. a. Dieser Abschnitt fällt mit der Ausbildung von Froststrukturen, besonders von Frostspaltennetzen in den Becken und mit der Bildung von lößartigen Sedimenten zusammen. Zur gleichen Zeit kommen Betula nana(?)-Pollen und dicht über diesem Horizont zahlreiche Blättchen von Dryas octopetala sowie von Zwergsträuchern vor. Selaginella selaginoides wurde nachgewiesen.

Im mittleren und oberen Bereich der Schluffmudde nimmt zunächst Betula, dann wieder Pinus zu. Diese 


\begin{tabular}{|c|c|c|c|c|c|c|c|c|c|c|c|c|c|c|c|c|c|c|c|c|c|c|c|}
\hline Probenummer & 75 & 73 & 71 & 69 & 67 & 65 & 63 & 61 & 59 & 57 & 55 & 53 & 51 & 49 & 47 & 45 & 43 & 41 & 39 & 37 & 35 & 33 & 31 \\
\hline Gehölze & 68,5 & 54,4 & - & 55,0 & 52,8 & 54,0 & 40,8 & 36,0 & 26,8 & 25,2 & 38,8 & 34,4 & 37,6 & 29,2 & 41,2 & 52,0 & 50,0 & 63,0 & 85,6 & 51,6 & 81,6 & 67,2 & 52,8 \\
\hline Kräuter/Gräser & 31,2 & 45,6 & - & 45,0 & 47,2 & 46,0 & 59,2 & 64,0 & 73,2 & 74,8 & 61,2 & 65,6 & 62,4 & 70,8 & 58,8 & 48,0 & 50,0 & 37,0 & 14,4 & 48,4 & 18,4 & 32,8 & 47,2 \\
\hline \multicolumn{24}{|c|}{ Sonstige (bezogen auf NB $+\mathrm{NBP}$ ) } \\
\hline Typha latifolia & - & - & - & + & + & + & - & - & - & - & - & + & - & - & - & + & 0,4 & 4,0 & 0,8 & 2,8 & 0,4 & - & - \\
\hline Typha-Sparganium & 0,4 & 0,4 & - & + & 0,4 & + & + & 0,4 & 0,4 & 0,4 & 1,2 & 0,8 & + & - & 0,4 & 2,0 & 0,8 & 1,0 & 0,8 & 0,8 & 0,4 & - & - \\
\hline Equisetum & - & - & - & - & - & - & - & 0,4 & 0,4 & - & - & + & + & - & - & - & + & 1,0 & + & 0,8 & 2,0 & + & - \\
\hline Nymphaea & - & + & - & - & - & - & - & - & - & - & - & - & - & - & - & - & - & - & + & 0,8 & + & - & - \\
\hline Ceratophyllum & - & - & - & - & - & - & 0,4 & - & - & 2,0 & 0,8 & 0,4 & + & 15,6 & 3,6 & 5,0 & 0,4 & - & + & - & + & - & - \\
\hline Potamogeton & - & - & - & - & - & - & - & 0,4 & - & - & 1,2 & - & - & 0,8 & 0,4 & - & - & - & - & - & - & - & - \\
\hline Myriophyllum spicatum & - & - & - & - & - & - & 0,4 & - & - & - & - & - & - & - & - & + & + & 6,0 & 0,8 & 0,4 & 0,4 & - & - \\
\hline Alisma plantago-aquatica & - & - & - & - & - & - & - & - & - & - & - & - & - &. & - & - & - & - & - & - & + & - & - \\
\hline Menyanthes & - & - & - & - & - & - & - & - & - & - & - & - & - & - & - & - & - & - & - & 0,8 & - & - & - \\
\hline Sphagnum & - & 0,4 & - & 2,0 & 0,4 & + & 0,4 & - & + & + & 1,2 & 1,6 & 0,4 & + & 0,4 & + & 0,8 & - & - & - & - & - & 0,4 \\
\hline Polypodiaceae & 18,4 & 2,4 & - & - & 1,2 & + & + & 0,4 & + & 0,4 & 1,6 & 0,8 & 0,8 & - & 0,4 & + & + & 1,0 & 0,4 & - & 0,4 & 0,8 & 0,4 \\
\hline Umgelagerte & 2,4 & 60,4 & - & 275,0 & 50,8 & 78,0 & 50,0 & 30,0 & 18,8 & 68,0 & 62,0 & 99,2 & 6,4 & 9,2 & 36,0 & 310,0 & 256 & 176 & 56,8 & - & 1,6 & 2,8 & 38,0 \\
\hline Pediastrum $b$ & - & 1,6 & - & 1,0 & 0,4 & 1,0 & 0,8 & 2,0 & 0,8 & 5,6 & - & 1,2 & 0,8 & 2,0 & 8,0 & 2,0 & 5,6 & 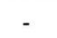 & - & 0,4 & - & - & \\
\hline Pediastrum kawraiskyi & - & - & - & - & - & - & - & - & - & - & 0,4 & - & - & - & - & - & - & - & - & - & - & - & \\
\hline Süßwasser-Plankter & 62,8 & 480,0 & - & 25,0 & 24,4 & 13,0 & 10,0 & 38,0 & 30,4 & 321 & 24,4 & 5,2 & 18,0 & 19,2 & 116 & 13,0 & 14,4 & 6,0 & 130 & 699 & 23,6 & 148 & 80 \\
\hline Moossporen & 0,4 & 12,0 & - & 1,0 & + & - & - & 1,6 & - & 1,2 & - & - & - & 0,8 & - & - & 1,2 & - & 0,4 & 3,2 & + & 1,2 & \\
\hline Pilzsporen & - & - & - & - & - & - & oft & oft & oft & oft & oft & - & oft & oft & oft & - & oft & - & oft & oft & - & - & - \\
\hline Dinoflagellaten & - & - & - & - & + & 1,0 & + & - & - & - & - & 0,4 & - & - & - & 2,4 & 1,0 & 0,8 & 5,2 & 0,4 & 2,4 & 2,4 & 0,8 \\
\hline Tiletia Spagnii & - & - & - & - & - & - & - & - & - & - & 0,4 & 0,4 & - & - & - & - & + & - & + & - & - & - & - \\
\hline Botryococcus & - & 0,8 & - & - & - & - & - & - & - & 0,8 & - & - & - & 0,4 & - & - & 0,4 & - & + & 0,8 & - & - & 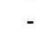 \\
\hline Anthoceras & - & - & - & - & - & - & - & - & - & - & - & - & - & - & - & - & - & + & - & - & - & - & 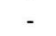 \\
\hline Sternhaare v. Nymphae & - & - & - & - & - & - & - & + & + & 0,8 & - & - & 0,4 & 0,4 & - & - & - & - & - & - & - & - & - \\
\hline Radizellen v. Cyperaceae & - & - & - & - & - & - & - & - & - & 0,4 & - & - & - & - & + & - & - & - & - & - & - & - & - \\
\hline Fussit & - & - & - & oft & - & - & oft & - & + & - & oft & oft & - & - & - & - & - & - & - & - & - & - & - \\
\hline Lignin-Reste & $\begin{array}{c}\text { sehr } \\
\text { oft }\end{array}$ & - & - & - & oft & - & - & - & - & - & - & - & - & - & - & - & - & - & - & - & - & - & 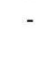 \\
\hline
\end{tabular}

in $\%$ + außerhalb des ausgezählten Bereichs beobachtet

erreicht Gipfelwerte von $50 \%$. Die Kräuteranteile bleiben etwa gleich hoch, während sich Gräser, vor allem Cyperaceae, stärker ausbreiten.

\subsubsection{Eichenmischwaldphase}

Im oberen Teil des Profils, der durch das Anmoor gebildet wird (Proben 75 bis 77), setzen plötzlich Thermophile des Eichenmischwaldes ein: Corylus, Quercus, Ulmus, Tilia, ferner Alnus, Picea und Carpinus. Hier ist bereits eine mittelholozäne Gesellschaft entwickelt. Der Boden zeigt einen längeren Hiatus an.

\subsection{Zur Vegetationsgeschichte}

Im allgemeinen entsprechen die einzelnen pollenanalytischen Abschnitte den für das Spätglazial nachgewiesenen Vegetationsphasen. Im Vergleich mit benachbarten Abfolgen aus dem Harzvorland (Ascherslebener/Gaterslebener See, Müller 1953, MaNia 1967) liegt eine gleichartige Sukzession vor. Es gibt Unterschiede, wohl von lokalem Charakter; unter ihrer Vernachlässigung entsprechen sich die Sukzessionsstufen wie folgt:

4.1.1 = Ic, 4.1.2 = IIa, 4.1.3 = IIb, 4.1.4 = III der Abfolge aus dem Ascherslebener See.
Die Ältere Dryas-Zeit (I c) wurde mit dem Basistorf erfaßt. Die Böllingschwankung (Ib) ist im Anmoor und in sandigen Mudden darunter nur mit Molluskenfaunen belegbar. Wie im Ascherslebener See ist die Ältere Dryas-Zeit durch hohe Salix- und Kräuterwerte, aber auch durch Hippophaë und Juniperus charakterisiert. Es waren Niederungstundren mit Strauch- und Mattenformationen entwickelt, in höheren Lagen breitete sich die Steppe aus.

Die Betula-Zeit nach dem Salix-Maximum gehört bereits in das Alleröd-Interstadial (IIa). Zunächst breiten sich Birkenwälder aus, die nach Aussage der Großreste als Gehölze in der Geiselaue wuchsen. Ihnen folgte die Ausbreitung von Kiefernwäldern und die dadurch charakterisierte Kiefernzeit (IIb). Allerdings zeichnet sich in der Ascherslebener Sukzession (MüLLER 1953) ein viel deutlicherer, einheitlicher Pinus-Gipfel mit über $60 \%$ Pollenwerten ab. Im Geiseltal wird dieser Gipfel durch lokal verursachte sehr hohe Gramineae-Werte unterdrückt; vielleicht eine direkte Folge des Staubniederschlags der LaacherSee-Eruption?

Während der Kurvenschnittpunkte von Pinus und Betula konnten Usinger (1985) in Norddeutschland und KNIPPING (1989) im Oberpfälzer Wald häufig PO- 


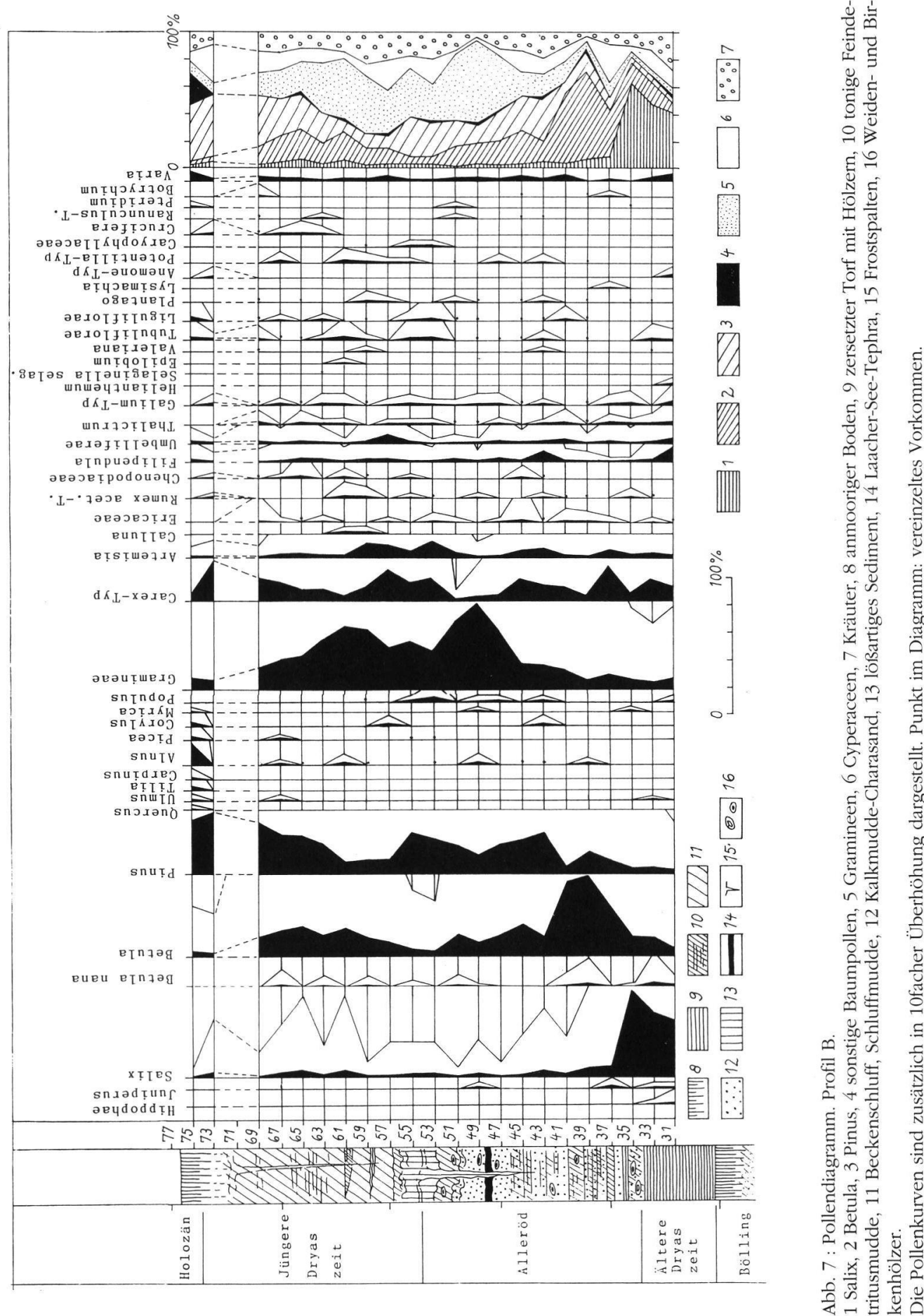

pulus beobachten. Im Traß der Umgebung des Laacher Sees kommen zahlreiche Reste, vor allem Blätter, von Populus tremula vor. Im Geiseltal-Profil war der schlecht erhaltungsfähige Pollen von Populus nicht immer eindeutig bestimmbar, aber die weni- gen sicheren Nachweise bestätigen sein Vorkommen auch hier. Populus war sicher ein wichtiger Baum in den allerödzeitlichen Gehölzen (KNIPPING 1989, USINGER 1985, RYBINIČKOVA 1974, JANKOVSKÁ 1980), auch im Geiseltal. 
Im Gegensatz zu anderen Diagrammen (z. B. UsiNGER 1985) ist Hippophaë im Geiseltalprofil selten. Er verschwindet mit dem Salix-Maximum. Das entspricht auch den Beobachtungen von MülLer (1953). Zu einer Auflichtung der Birken- und Kiefernwälder kam es während der Jüngeren Dryas-Zeit (III). Damit verbunden war wieder eine Zunahme der Vegetation offener Landschaftstypen, allen voran von $A r$ temisia sowie von Strauchgesellschaften. Steppen und Niederungstundren weiteten sich wieder aus. Das wird auch im Sedimentwechsel bemerkbar.

Im Verlaufe der Jüngeren Dryas-Zeit setzte wie im Nordharzvorland wieder die Ausbreitung von Birken- und Kiefernwäldern ein. Besonders die Kiefer gewinnt gegen Ende der Phase an Bedeutung. Anteile der offenen Landschaft bleiben jedoch erhalten.

\section{Die Molluskenfauna und ihre Lebensräume (Mania, D.)}

Das Spätglazial als eine Zeit allgemeiner Wiedererwärmung und damit verbundener Auflösung des Dauerfrostbodens rief eine ständig zunehmende Vernässung der Niederungen und Talauen, besonders während der wärmeren Phasen, hervor. Dadurch ist die Molluskenentwicklung im Profil des Geiseltals gekennzeichnet (s. Abb. 8 bis 10.)

In den wärmeren Phasen überwiegen Sumpf- und Gewässerfaunen, während in den kaltklimatischen Ablagerungen terrestrische Gesellschaften der offenen Landschaft dominieren.

Allgemein läßt sich vom Hochglazial bis zum Holozän eine Zunahme der Artenhäufigkeit und, abgesehen vom allgemeinen Ablauf, ein qualitativer Wechsel beobachten. Er besteht darin, daß die hochglaziale Lößsteppenfauna, die sog. Pupilla-Fauna (LOŽEK 1964, 1965, MANIA 1973) von der für feuchtere Verhältnisse der Tundren typischen ColumellaFauna abgelöst wird. Gegen Ende der Jüngeren Dryas-Zeit verschwinden dann endgültig die kennzeichnenden Arten dieser Faunen. In den wärmeren Phasen gewinnen Assoziationen feuchter und mikroklimatisch begünstigter Standorte immer mehr an Bedeutung. Sie gehören allgemein zum Grundbestand von Faunen, die für Waldsteppenverhältnisse typisch sind. Sie ähneln der Arianta- und Discus ruderatus-Fauna (LožEK 1964, 1965), deren Vertreter ab Bölling in der Geiseltalfauna vorhanden sind. Discus ruderatus als anspruchslose boreo-alpine Waldart tritt jedoch erst im Alleröd in den benachbarten Hügel- und Bergländern des mittleren Saaletales, der Hainleite und des Unterharzes auf und kennzeichnet damit diese Landschaften als die hauptsächlichen Ausbreitungsgebiete der allerödzeitlichen Taiga (Mania 1972). Im mitteldeutschen
Trockengebiet herrschten neben den sich ausbreitenden Gehölzen noch an Artemisia, Chenopodiaceen und Gramineen reiche Wiesensteppen vor. Am Ende des Spätglazials verschwinden alle an kaltklimatische Verhältnisse angepaßten Arten. Im Frühholozän sind boreo-alpine Arten, die das Spätglazial charakterisierten, noch wichtige Faunenelemente. Sie verschwinden allmählich im Verlaufe des Boreals. Statt dessen treten immer mehr thermophile Arten auf. Seit dem jüngeren Atlantikum wird die warmzeitliche Faunenentwicklung wie die Landschaft immer mehr durch den anthropogenen Eingriff in den Naturhaushalt empfindlich verändert (MANIA 1973).

\subsection{Hochglazial}

Die Faunen aus Löß und Beckenschluffen sind ausgesprochen artenarm und anspruchslos wie auch unempfindlich gegen Kälte und Trockenheit und gegen hohe Temperaturschwankungen. Sie besiedelten die z. T. kräuterreichen, z. T. durch Gräser gekennzeichneten Lößsteppen unter ausgesprochen kalt-ariden Bedingungen. Sie bestehen vorwiegend aus Pupilla muscorum, deren forma densegyrata, Pupilla loessica, Trichia hispida und Succinea oblonga, in einem Falle sogar nur aus Pupilla muscorum und Succinea oblonga. Das ist der Grundbestand der Pupilla-Fauna. In den Beckenlößen verweisen einige akzessorische Elemente auf Sümpfe und periodische Kleingewässer unter den Bedingungen des arktischen Sommers (Sphaerium corneum, Lymnaea truncatula, Pisidien). Das Klima war arktisch/hocharktisch, trocken.

\subsection{Müchelner Intervall 1 (Profil A)}

Insgesamt kommen 10 Arten vor (ohne Pisidien). Der Bestand der hochglazialen Pupilla-Fauna bleibt erhalten. Dazu kommen einige Arten, die ebenfalls für kaltzeitliche Verhältnisse typisch sind, wie die heute in nordasiatischen Grasländern und Gebirgssteppen lebende Vallonia tenuilabris, sowie einige Sumpfarten, die in temporären, pflanzenreichen Kleingewässern während des subarktischen Sommers leben können (Lymnaea peregraf. ovata, Lymnaea truncatula, Gyraulus acronicus rossmaessleri, Anisus leucostomus, Sphaerium corneum, verschiedene Pisidien). Die Pupilla-Fauna deutet auf Steppen hin, die Begleitfauna auf Sümpfe in den Auen und auf Niederungstundren an weniger feuchten Stellen mit Matten und Strauchgesellschaften. Das geht auch aus den pflanzlichen Resten der Mudde hervor. Sie stammen von Carex-Arten, von Sträuchern und von Ranunculus aquatilis und Myrio- 
phyllum verticillatum, letztere beiden als Vertreter der Kleingewässer. Das Klima war subarktisch, trocken.

\subsection{Kaltphase zwischen den Müchelner Intervallen 1 und 2}

Aus dem Beckenschluff des Profils B stammt eine Thanatozönose. Sie besteht aus 9 Arten, die z. T. der Pupilla-Fauna, z. T. der jetzt erstmalig in Erscheinung tretenden Columella-Fauna angehören. Der typische Vertreter dieser Fauna ist die heute in hohen nördlichen Breiten $\left(67-71^{\circ} \mathrm{N}\right.$. Br.) und in den alpinen Zonen der Hochgebirge in feuchten bis sumpfigen Wiesen, auf steinigem Untergrund in der niederen inselartigen Vegetation, an Felsen und in nassen subarktischen Wäldern lebende Columella columella. In ihrer Begleitung treten eurytherme Arten auf, die an feuchte Verhältnisse angepaßt sind, wie Succinea putris. Doch herrschen in der vorliegenden Thanatozönose noch die Arten der Pupilla-Fauna vor ( $P$. muscorum, $P$. $m$. densegyrata, $P$. loessica, Vallonia tenuilabris, Succinea oblonga, Trichia bispida). Sie erreichen noch etwa $80 \%$. Eine Steppenform bereichert diese Gesellschaft: Pupilla sterri. Das Klima war arktisch, trocken.

\subsection{Müchelner Intervall 2 (Profile A und B)}

Die Columella-Fauna wird in diesem Intervall zur beherrschenden Komponente. Insgesamt kommen 24 Arten vor (ohne Pisidien). Neben C. columella (bis $27 \%$ ) erreichen Pupilla muscorum und Vallonia tenuilabris höchste individuelle Häufigkeitswerte. Andere, für trockene Steppen typische Arten, fehlen oder sind nur mit geringen Anteilen vertreten. Häufig werden jetzt eurytherme Arten mit verschiedenen Feuchtigkeitsansprüchen, z. B. Cochlicopa lubrica, Punctum pygmaeum, Perpolita radiatula, Euconulus fulvus, Succinea'putris und Succinea oblonga. Sie deuten noch mehr als die artenarme Fauna des Intervalls 1 auf ausgedehnte Carex-Wiesen und -sümpfe, feuchte Matten- und Strauchfluren hin. In den Strauchfluren konnte auch die vorwiegend alpine Art Eucobresia diaphana leben. Als Gewässerarten erscheinen Lymnaea peregra $\mathrm{f}$. ovata, Physa fontinalis und Armiger crista. Sie verweisen auf kleine stehende, bleibende und sommerwarme Gewässer - eine Tendenz, die sich schon im vorangehenden Intervall andeutet. Die Gewässer waren pflanzenreich. Neben Carex-Früchten kamen solche von Myriophyllum verticillatum zum Vorschein. Lymnaea truncatula und Anisus leucostomus lebten in sumpfigen Kleingewässern oder im versumpften Uferbereich der Flachgewässer. Unter den Arten der offenen Landschaft treten in der Suk- zession erstmalig seit dem Hochglazial Vallonia costata und Vallonia pulchella auf. Sie stellen höhere Ansprüche an ihre Umwelt. Sie fehlen daher in kaltklimatischen Assoziationen. Außerhalb der feuchten, pflanzenreichen Niederungen bestanden noch weiterhin kräuterreiche Grassteppen - der Lebensraum auch für diese Arten. Das Klima war subarktisch, relativ trocken.

\section{5 Älteste Dryas-Zeit}

Bis 23 Arten (ohne Pisidien) konnten in den Beckenschluffen und Sandmudden mit reichlich Ast- und Zweigdetritus von Sträuchern festgestellt werden. 8 bis 20 Arten setzten jeweils die Gesellschaft zusammen. Vertreter der Pupilla- wie der Columella-Fauna bilden den Grundbestand. Sie erreichen bis über $60 \%$ des individuellen Anteils. In feuchtigkeitsliebenden Assoziationen hat C. columella mit akzessorischen Elementen das Übergewicht. Allein mit Succinea putris beansprucht sie $55 \%$ des individuellen Bestands. Handelt es sich um trockene Standorte, dann treten sofort die Arten der Pupilla-Fauna auf: alle bisher genannten Pupilla-Arten, vor allem Pupilla loessica, dazu Succinea oblonga und Vallonia tenuilabris.

Auf Kleingewässer, überwiegend periodische Sümpfe und Wasserstellen, verweisen einige Wasserarten. In der Aue dehnte sich eine Niederungstundra aus, an trockenen Stellen, aber viel mehr auf den benachbarten Hochflächen, die Grassteppe. Das Klima war arktisch, trocken.

\subsection{Bölling-Interstadial}

Aus verschiedenen organogenen Sedimenten stammt die Fauna mit insgesamt 34 Arten (ohne Pisidien). 11 bis 30 Arten setzen die jetzt schon relativ artenreichen Gesellschaften zusammen. In der Landfauna überwiegt noch die Columella-Fauna mit allen ihren Vertretern, zusätzlich aber mit Vallonia costata und Vallonia pulchella. Pupilla muscorum und Vallonia costata sind am häufigsten. Typische Arten der kaltzeitlichen Fauna (C. columella, Vallonia tenuilabris, $P$. m. densegyrata) treten in den Hintergrund oder fehlen ( $P$. loessica). Aber zahlreiche, etwas anspruchsvollere feuchtigkeitsliebende Arten kommen hinzu, wie Cochlocopa lubrica, Punctum pygmaeum, Succinea putris und die boreo-alpinen Formen von Vertigo genesii, die einschließlich des ebenfalls boreo-alpinen Gyraulus acronicus, der die Gesellschaften stehender, pflanzenreicher Kleingewässer kennzeichnet, zu charakteristischen Elementen der Fauna werden. In den Gewässerfaunen tritt erstmalig Acroloxus lacustris auf. Im Verein mit 


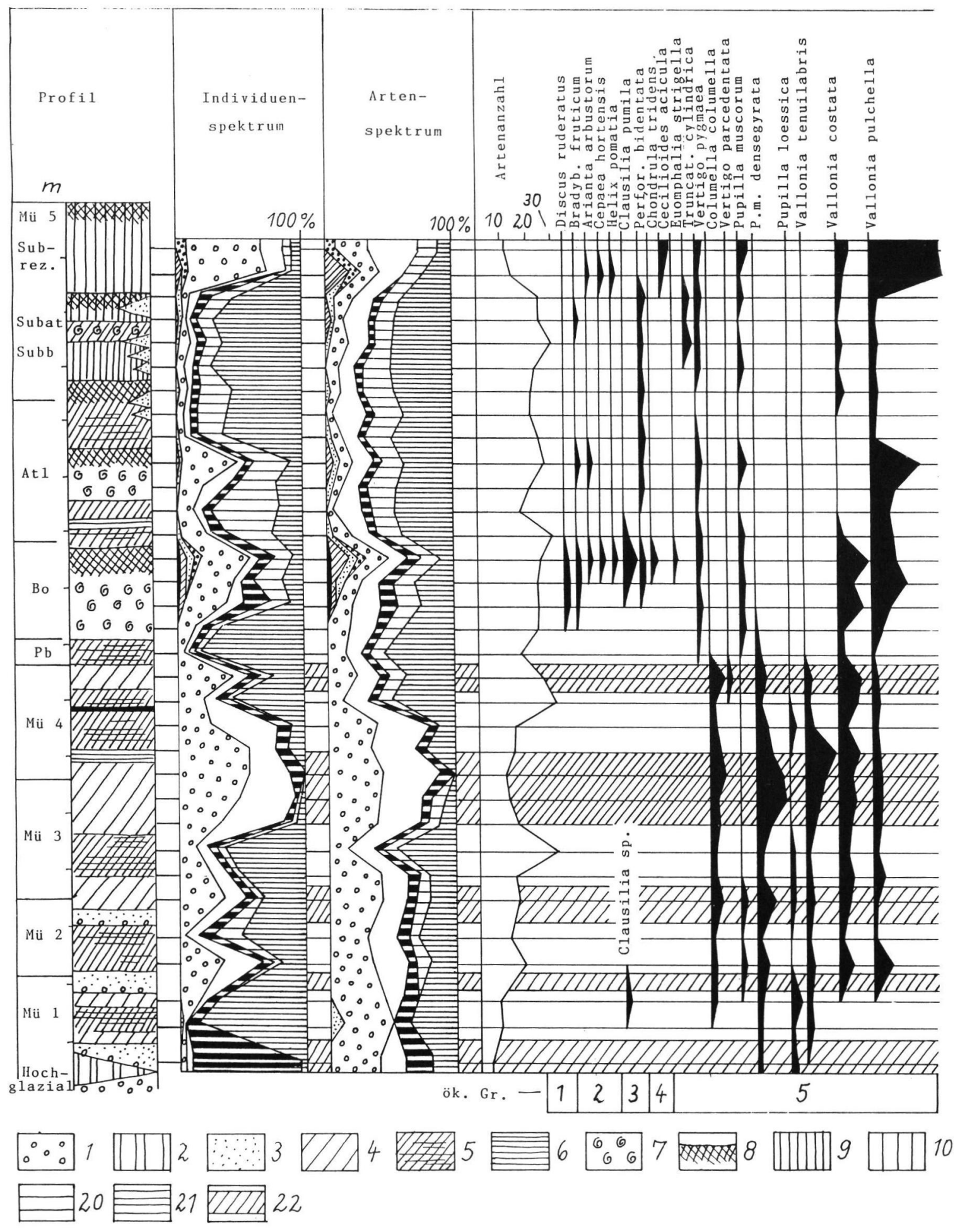

Abb. 8: Spät- und postglaziale Molluskensukzession. Profil A.

1 sandige Kiese, 2 Löß, 3 Sand, 4 Beckenschluff, 5 Schluff- und Tonmudden, Feindetritus-, Grobdetritusmudden, 6 Torfe,

7 Kalkmudden, Seekalk, 8 humoser Boden, 9 unterer humoser Auelehm, 10 oberer Auelehm, 11 Laacher-See-Tephra,

12 Denudationsfläche, 13 - 21 Arten- und Individuenspektrum: 13 Waldarten, 14 Arten vorwiegend im Wald lebend, im 


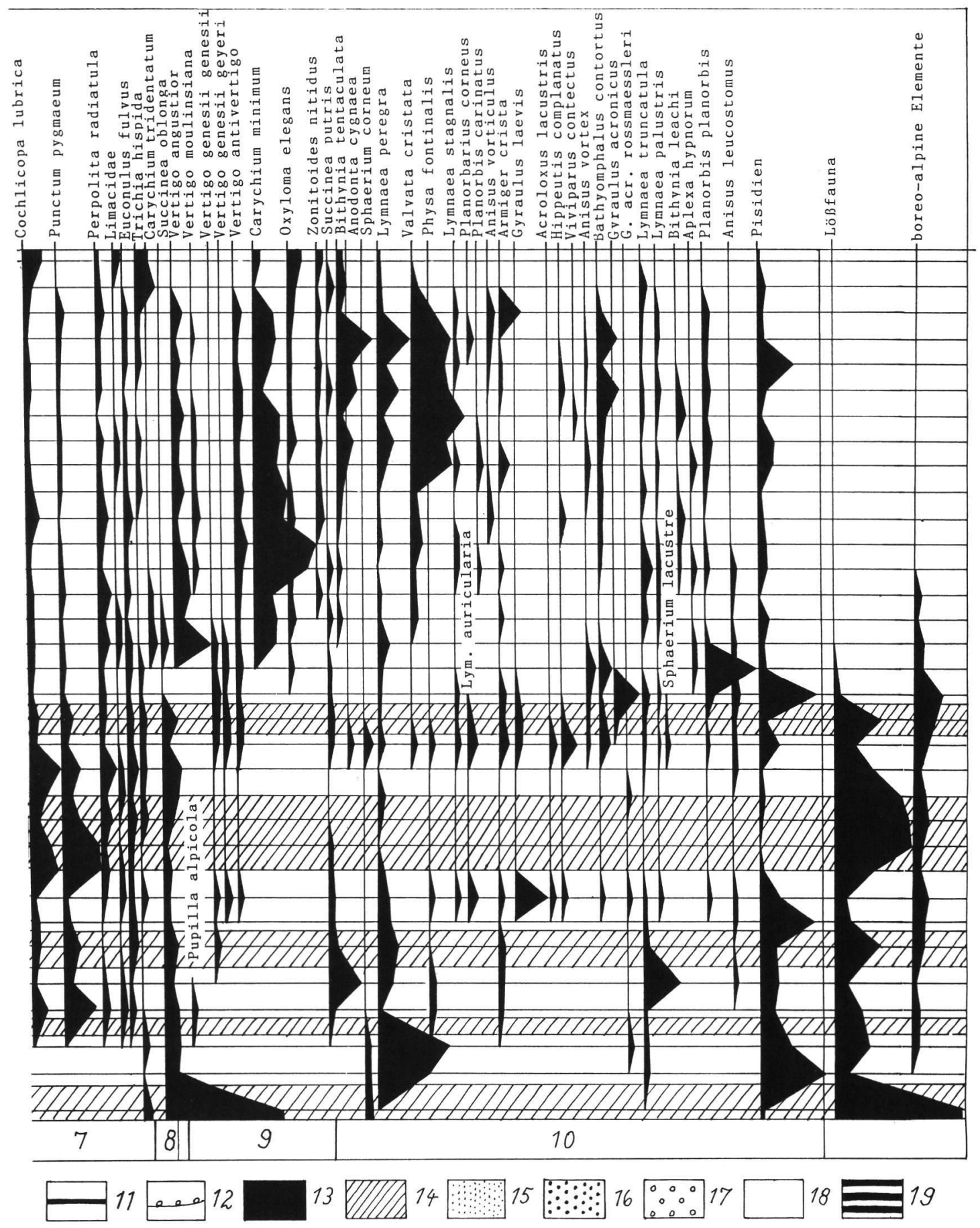

Gebüsch und in der Waldsteppe, 15 Auwaldarten, 16 Steppenarten, 17 allgemeine Arten der offenen Landschaft, 18 mesophile Arten, überwiegend euryök, 19 Arten mit höheren Feuchtigkeitsansprüchen, 20 Sumpfarten, 21 Wasserarten, 22 im Diagramm die kaltklimatischen Abschnitte. 


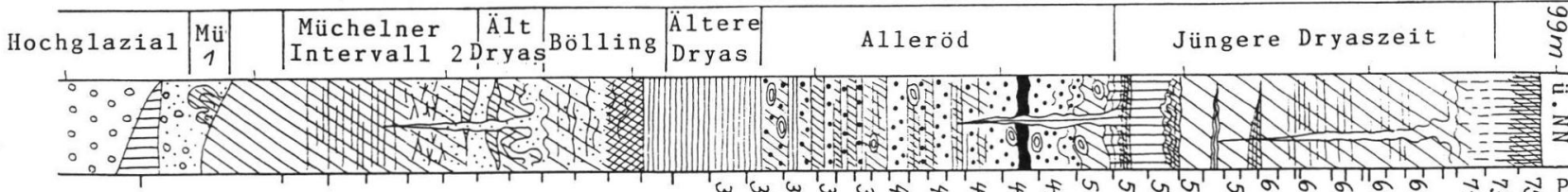

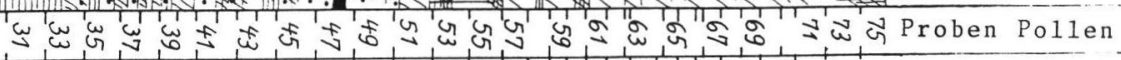

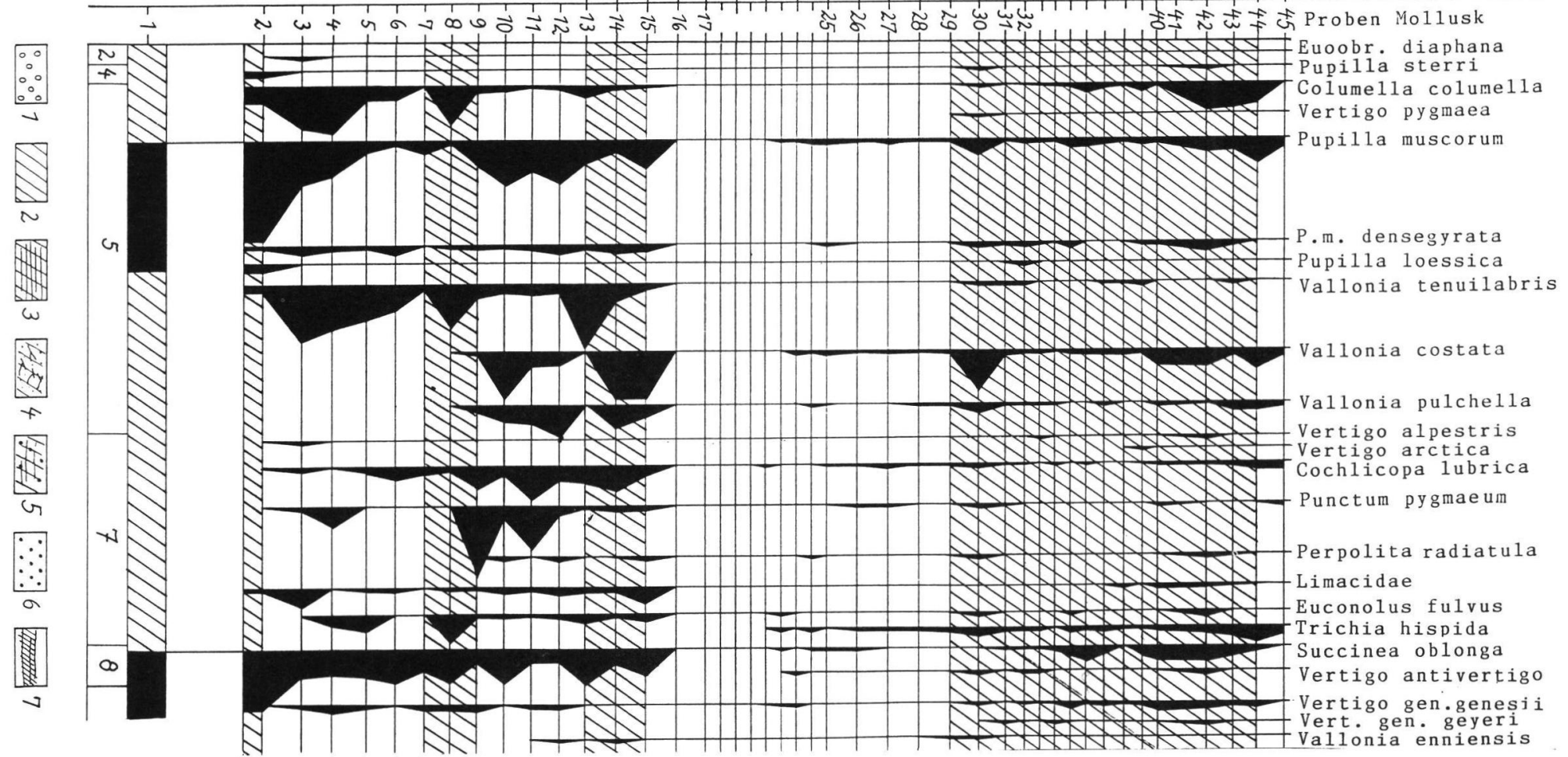




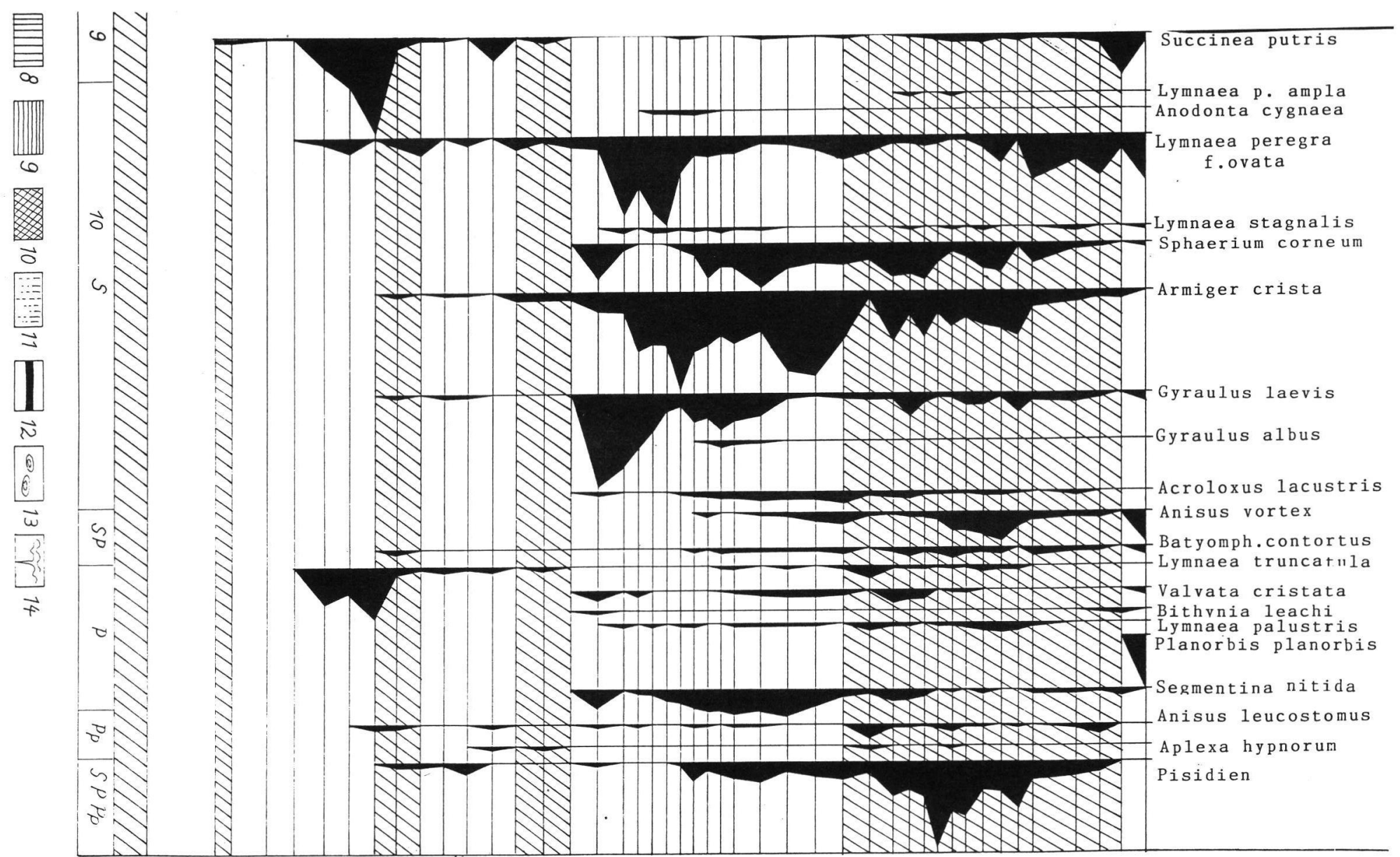

Abb. 9 Spätglaziale Molluskensukzession. Profil B.

1 sandige Kiese, 2 Beckenschluff, Schluffmudde, 3 Schluff- und Tonmudden, Feindetritus-, Grobdetritusmudden, 4 Muddesand mit Grobdetritus aus Sträuchern, 5 Wechsellagerung von toniger Feindetritusmudde und Kalkmudde/Charasand, 6 Kalkmudde/Charasand, 7 fein gewarvter Schluff, 8 lößartiges Sediment, 9 Torfe, 10 Anmoorboden, 11 Bleichhorizont, 12 Laacher-See-Tephra, 13 Birken- und Weidenhölzer, 14 Froststrukturen.

2 - 10, S, SP, P, Pp - ökologische Gruppen und ökologische Kennzeichnung - siehe Tab. 2 


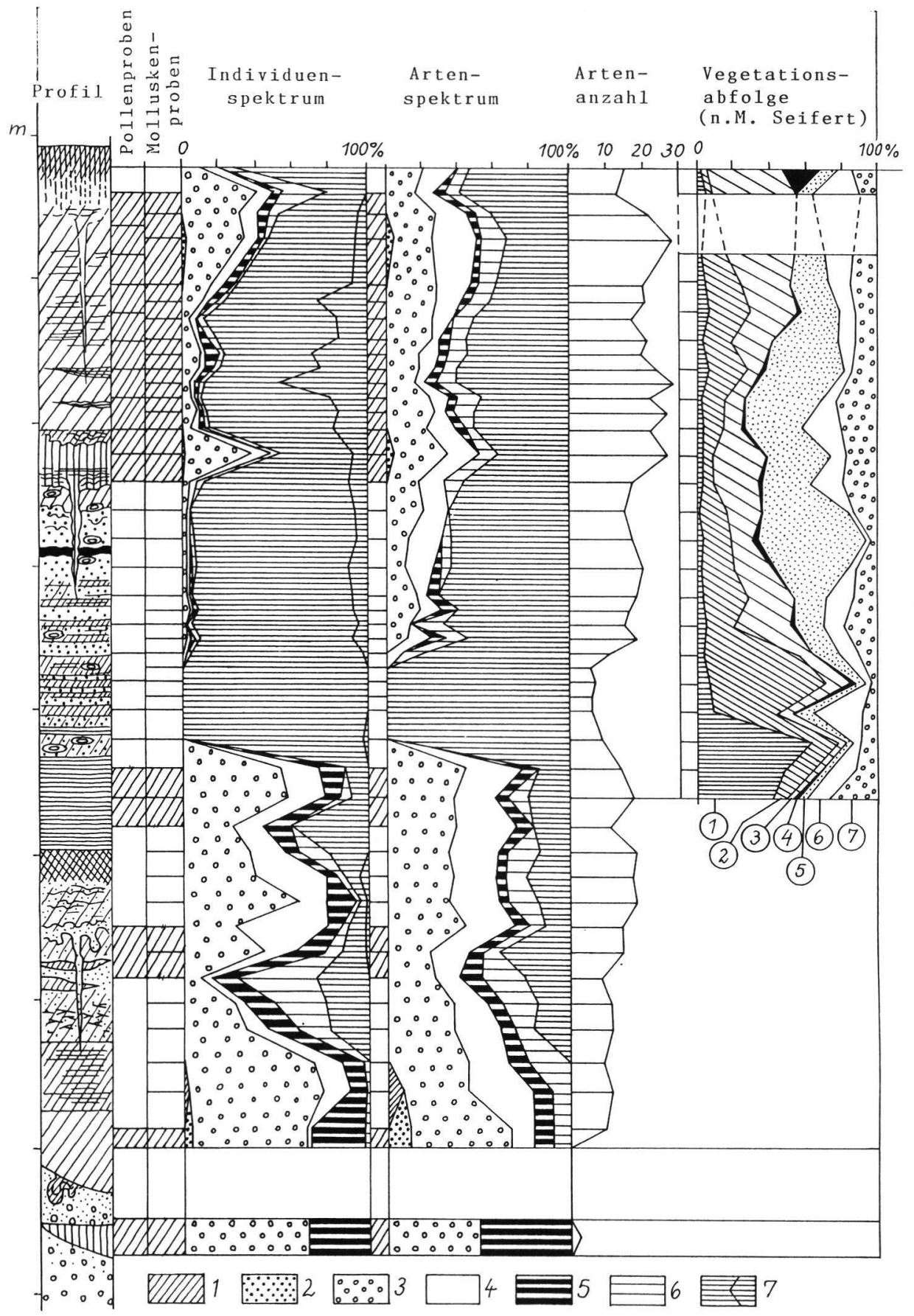

Abb. 10: Spätglaziale Molluskensukzession im Vergleich mit der Vegetationsentwicklung. Profil B. Erklärung des Profils: siehe Abb. 9.

Ökologische Gruppen: 1 Arten der Waldsteppe, 2 Arten der Steppe, 3 allgemeine Arten der offenen Landschaft, 4 euryöke, mesophile Arten, 5 Arten mit hohen Feuchtigkeitsansprüchen (ausgenommen Succinea oblonga als Lößart), 6 Sumpfarten, 7 Wasserarten, rechts: Pisidien.

Gruppen der Vegetationsabfolge: 1 Salix, 2 Betula, 3 Pinus, 4 sonstige Bäume, 5 Gramineen, 6 Cyperaceen, 7 Kräuter. 
Pflanzenresten lassen sich in der Geiselniederung als Lebensräume flache pflanzenreiche, eutrophe Wasserbecken, Carex-Sümpfe, durchnäßte Moosrasen, feuchte Wiesen, dichte z. T. sumpfige Strauchfluren, besonders aus Weiden sowie erste Birkengehölze rekonstruieren. Außerhalb dieser Standorte herrschte weiterhin die jetzt aber mehr und mehr sommerwarme Grassteppe. Das Klima war bereits über den subarktischen Bereich hinaus entwickelt und etwas feuchter.

\section{7 Ältere Dryas-Zeit}

Aus relativ mächtigen Beckenschluffen (Profil A) und in verschiedenem Grade zersetzten Moos- und Grastorfen (Profil B) stammen Molluskengesellschaften aus 8 bis 17 Arten. Insgesamt umfaßt die Fauna 30 Arten. Wieder überwiegen die Faunen der offenen Landschaft, vor allem der Columella-Fauna mit zahlreichen Offenlandarten und Arten verschiedener Feuchtigkeitsansprüche. Auch Vertreter der Pupilla-Fauna rücken wieder in den Vordergrund, z. B. Pupilla loessica. Vallonia costata, die konstant seit Bölling nachgewiesen werden kann, deutet darauf hin, daß ausgesprochen arktische Verhältnisse nicht mehr erreicht werden. Insgesamt nehmen die Offenlandarten mehr als $50 \%$ des individuellen Anteils der Fauna ein. Diese ist im Profil B mit dem Salix-Gipfel der Pollensukzession assoziiert (vgl. Abb. 7, 10) und hinsichtlich ihrer zahlreichen Arten der Steppe und Tundra mit ebenfalls hohen Anteilen von Gramineen und Kräutern sowie dem Auftreten von Hippophaë und Juniperus verbunden. Großreste und Früchte und Samen von Sträuchern, CarexArten, Moosen deuten ebenfalls auf die Strauchfluren, Carex-Sümpfe, Moosrasen, Wiesen und Matten in der vor allem sommerlich durchnäßten Aue und auf die Artemisia-Grassteppen der benachbarten Hochflächen hin. Das Klima war arktisch bis subarktisch und relativ trocken.

\subsection{Alleröd-Interstadial}

Mit insgesamt 44 Arten wird das erste sehr artenreiche Artenspektrum nach dem Hochglazial erreicht. Neben artenreichen Gesellschaften mit 30 bis 35 Arten erscheinen auch ausgesprochen artenarme, an besondere Standortbedingungen geknüpfte Gesellschaften aus 6 bis 11 Arten. Es kommt die gesamte Bölling-Fauna vor, ihr treten einige anspruchsvollere Einwanderer zur Seite, so daß eine neue Sukzessionsstufe erreicht wird.

$\mathrm{Zu}$ den artenarmen Gesellschaften gehört die Gewässerfauna (z. B. Profil B). Die Birkenzeit (IIa) ist besonders durch hohe Anteile von Lymnaea peregra f. ovata und Gyraulus laevis, die Kiefernzeit (IIb) statt dessen von Armiger crista, Sphaerium corneum, Acroloxus lacustris und Segmentina nitida charakterisiert. Die IIa-Fauna kann als Bölling-Assoziation aufgefaßt werden, die sich zur IIb-Fauna weiterentwickelt. Dabei treten während des Klimaoptimums in IIb weitere anspruchsvollere Arten auf, wie Gyraulus albus, Anodonta cygnaea, Anisus vortex. Die Artenarmut ist durch die besonderen Bedingungen des von Characeen durchwucherten kalkhaltigen Gewässers verursacht. Ein dichter Vegetationsfilter am sumpfigen Ufer aus Carex-, Typha-, Strauch- und Birkenbeständen verhinderte, daß die Landfauna in größeren Mengen in die Wasserbecken gelangen konnte. Aber wir kennen die Landfauna aus den benachbarten Aufschlüssen (z. B. Profil A). Es herrschte nach wie vor die Fauna der offenen Landschaft, aber mit einem Übergewicht jener Arten, die nicht zur Kaltzeit-Fauna gehören, z. B. von Vallonia costata, V. pulchella und Vertigo pygmaea in Gemeinschaft mit Pupilla muscorum und wechselnd hohen Anteilen zahlreicher Arten mit verschiedenen Feuchtigkeitsansprüchen. Diese Fauna besiedelte ebenso gut die lichten Wälder an feuchten bis trockenen Standorten. Aber echte Waldarten fehlen hier; sie treten in Gestalt des Discus ruderatus zur gleichen Zeit in den benachbarten Bergländern auf (MANIA \& STECHEMESSER 1969).

Im Alleröd sind noch alle Arten der Columella-Fauna vorhanden (s. Tab. 2). Die Pupilla-Fauna fehlt mehr oder weniger.

Die feuchten bis nassen Standorte beherbergten Sumpffaunen mit Succinea putris, Vallonia enniensis und den neu auftretenden Arten Vertigo antivertigo und Vertigo angustior. Zusätzlich kommen noch boreo-alpine Elemente vor (Vertigo genesii, Pupilla alpicola). Die Niederung war stark durchnäßt und von größeren pflanzenreichen Wasserbecken und ausgedehnten Sümpfen mit Carex- und Typha-Beständen, mit Strauchdickichten aus Weiden, mit Birkengehölzen in Form von Bruchwäldern bedeckt. Kiefernreiche Gehölze kamen erst außerhalb der Niederung vor, dort breiteten sich jedoch noch weitflächig Grassteppen aus. Nach Auskunft eines durch die Laacher Tephra datierten Bodenprofils am Ascherslebener See (Weinberg bei Schadeleben, Altermann \& Mania 1968) waren sie sommerwarme Landschaften, in denen die TschernosemBildung begann. Sie hatten demnach schon den Charakter von Tschernosem-Wiesensteppen. Aussagen von Fauna, Vegetation und Sedimenten stimmen bei den vielfältig untersuchten Geiseltalprofilen gut überein. Das Klima während des Höhepunktes war kühl-temperiert und relativ feucht (im Gegensatz zum vorausgehenden Teil des Spätglazials). 
Tab. 2: Spätglaziale und holozäne Molluskenfaunen aus dem Geiseltal

Ökolog. Gruppen

\begin{tabular}{rlr}
\hline $1 \mathrm{~W}$ & Discus ruderatus & + \\
\hline \multirow{2}{*}{$2 \mathrm{~W}(\mathrm{M}) \quad$} & & \\
& & \\
& Eucobresia diaphana & \\
& Bradybaena fruticum & + \\
& $(+)$ Arianta arbustorum & + \\
& ! Cepaea hortensis & + \\
(WS) ! Cepaea nemoralis & + \\
& & + \\
\hline
\end{tabular}

$3 \mathrm{~W}(\mathrm{~h})$

\section{Clausilia pumila}

$+$

(+) Perforatella bidentata

$4 \mathrm{~S}$

+ Pupilla sterri

(+) Chondrula tridens

(+) Helicopsis striata

! Cecilioides acicula

\begin{tabular}{|c|c|c|c|c|c|}
\hline $5 \mathrm{Ws}$ & & Euomphalia strigella & + & & \\
\hline $\mathrm{O}$ & ++ & Columella columella & + & ++++ & ++ \\
\hline & & Truncatellina cylindrica & + & & \\
\hline & & Vertigo pygmaea & + & + & \\
\hline & ++ & Vertigo parcedentata & & & + \\
\hline & + & Pupilla muscorum & ++ & ++++ & ++++ \\
\hline & ++ & P.m. densegyrata & & ++++- & ++++ \\
\hline & ++ & Pupilla loessica & + & +++ & ++++ \\
\hline & ++ & Vallonia tenuilabris & + & ++++ & ++ \\
\hline & $\mathrm{W}(+)$ & Vallonia costata & ++ & ++++ & + \\
\hline & & Vallonia pulchella & ++ & ++++ & + \\
\hline
\end{tabular}

$6 \mathrm{X}$

Cochlicopa lubricella

\begin{tabular}{cc}
$7 \mathrm{Mf}$ & \multicolumn{1}{c}{ Vertigo alpestris } \\
$\mathrm{M}$ & + Vertigo arctica \\
& $(+)$ Cochlicopa lubrica \\
& $(+)$ Punctum pygmaeum \\
$(+)$ Perpolita radiatula \\
$(+)$ Limacidae \\
$(+)$ Euconulus fulvus \\
+ Trichia hispida
\end{tabular}

$+++$

$+$

$+$

$+$

$+$

1 Holozän, 2 Jüngere Dryas-Zeit, 3 Alleröd, 4 Ältere Dryas-Zeit, 5 Bölling, 6 Älteste Dryas-Zeit, 7 Müchelner Intervall 2 , 8 Kaltphase zwischen Intervall 1 und 2, 9 Müchelner Intervall 1, 10 Hochglazial

Ökologische Gruppen (nach LOŽEK 1964): 1 Waldarten, 2 vorwiegend im Wald lebende Arten, auch in Gebüsch, Waldsteppe, 3 Waldarten mit hohen Feuchtigkeitsansprüchen, Auwald, 4 Steppenarten, 5 waldfeindliche Arten, allgemein offene Landschaft, 6 trockenheitsliebende Arten, 7 euryöke Arten an mittelfeuchten Standorten, 8 an feuchten Standorten, 9 mit hohen Feuchtigkeitsansprüchen, Sümpfe, 10 Wasserarten. - Ökologische Kennzeichnung: W Wald, M mittelfeuchte Standorte, h hygrophil, Ws Waldsteppe, S Steppe, O offene Landschaft allgemein, X xerotherm, f an Felsen, H feuchtigkeitsliebende Arten, P Sumpfarten, S stehendes Wasser, F fließendes Wasser, Pp periodische Sümpfe, ! warmzeitliche Leitarten, ++ kaltzeitliche Leitarten, im Löß, + im Löß, (+) auch im Löß. 


\subsection{Jüngere Dryas-Zeit}

An zahlreichen Stellen konnten aus Beckenschluffen und Schluffmudden Proben entnommen werden. Die spezielle Entwicklung der Fauna zeigt das mächtige Profil B. Insgesamt treten 45 Arten auf (ohne Pisidien). Die Bestände schwanken zwischen 8 und 27 Arten. Gleich zu Beginn der Zeit, verbunden mit Pinus-Rückgang und Rückkehr hoher Werte von Salix-, Kräuter- und Gräserpollen treten auch Columella- und Pupilla-Fauna mit ihren kennzeichnenden Vertretern wieder auf, besonders in jenem durch Löß und Froststrukturen gekennzeichneten Horizont. Offenlandarten erreichen bis $40 \%$ der Fauna. Sie zeigen die Wiederkehr tundren- und steppenartiger Verhältnisse an. Doch bleiben einige anspruchsvollere Arten zurück, wie Vallonia costata und $V$. pulchella. Sie deuten auf weniger extreme kaltzeitliche Verhältnisse hin, wie auch das konstante Vorkommen individuenreicher Komponenten mit meso- bis hygrophilen Arten wesentlich feuchtere Verhältnisse anzeigt als in den vorangegangenen Kaltphasen. Aber die meisten besonders anspruchsvollen Arten der Allerödfauna fehlen. Sie kehren erst im Frühholozän zurück. Die klimatischen Verhältnisse müssen ausgereicht haben für lokale Lößverwehung und -bildung, für das Entstehen von Frostspaltennetzen und von Flugsanddecken, wie sie am Ascherslebener See nachgewiesen werden konnten (MANIA 1967).

Die weitere Entwicklung, verbunden mit erneuter Birkenwälder-Ausbreitung und nachfolgendem, zögerndem Vordringen der Kiefer, führte wieder zu stehenden Flachgewässern mit schwach organogener Sedimentbildung und Molluskengesellschaften, die durch hohe Anteile einer Pisidium-Sphaerium corneum-Assoziation gekennzeichnet sind. Aus ihnen entwickelten sich während der weiteren Ausbreitung der Kiefer artenreiche Gesellschaften, die dem Sukzessionsstadium der Bölling-Zeit ähneln (mit Lymnaea peregra, Anisus leucostomus, Anisus vortex, Armiger crista, Gyraulus laevis). Während der gesamten Jüngeren Dryas-Zeit bleibt die ColumellaFauna erhalten, selbst gegen ihr Ende, während der Kiefern-Ausbreitung, kommen noch Vallonia tenuilabris und Columella columella, diese mit bis zu $15 \%$ Anteil, in der Fauna vor. Zum letzten Male treten auch Arten der Pupilla-Fauna auf, wie Pupilla muscorum densegyrata und Pupilla loessica. Im Vergleich mit allen anderen Untersuchungsergebnissen zeigt auch die Molluskenfauna die Existenz von Baum- und Strauchtundren mit weitflächig in der Niederung verbreiteten Carex-Sümpfen und -Wiesen sowie Grassteppen auf den Hochflächen an. Das Klima war arktisch-subarktisch, relativ feucht.

\subsection{Holozän}

Die Fauna ist am artenreichsten (60 Arten) und durch das Einwandern der thermophilen Arten gekennzeichnet. Wie auch an anderen Stellen des mitteldeutschen Trockengebiets entwickelt sich hier jedoch keine typische Waldfauna. Lediglich Auwaldkomponenten sind vorhanden, die während des Boreals durch den boreo-alpinen Discus ruderatus gekennzeichnet sind (MANIA 1972, Mania \& TOEPFER 1971). Thermophile Arten sind z. B. Helix pomatia, Cepaea hortensis, Cepaea nemoralis. Auffällig ist das Vorkommen der meridionalen Art Vertigo moulinsiana, die heute unserer Fauna fehlt, im Geiseltal nach Aussage archäologisch datierter Horizonte erst nach dem 15. Jahrhundert mit Beginn der "Kleinen Eiszeit" verschwand. Die frühholozäne Fauna ist noch - wie im Alleröd - durch boreo-alpine Arten gekennzeichnet (Vertigo genesii, Gyraulus acronicus). Im gesamten Holozän hat im Geiseltal die Fauna der offenen Landschaft große Bedeutung. Die Steppenarten Chondrula tridens und Helicopsis striata werden jetzt zu kennzeichnenden Elementen. Nach der Störung der natürlichen Sukzession durch den Menschen seit dem Neolithikum wird diese Komponente der offenen Landschaft zur vorherrschenden Fauna. Zusätzlich wandern in diese Fauna Kulturfolger ein, wie z. B. die süd- und südosteuropäischen Steppenschnecken Cecilioides acicula, Helicella obvia, Helicella itala und später auch Cernuella neglecta. Entsprechend der Vielfalt an Standorten im Geiseltal und ihrem stratigraphischen Wechsel ist auch die Molluskenfauna vielfältig zusammengesetzt und verweist mit ihren Komponenten auf verschiedenste Gewässer, Sümpfe, Auewälder, feuchte und trockene Wiesen, Gebüsche, Parklandschaften, Hügelsteppen und die spätere Kulturlandschaft (vgl. ManIa \& TOEPFer 1971, MANIA 1972, 1973).

\section{Schriftenverzeichnis}

Altermann, M. \& Mania, D. (1968): Zur Datierung von Böden im mitteldeutschen Trockengebiet mit Hilfe quartärgeologischer und urgeschichtlicher Befunde. Thaer-Archiv, 12: 539 - 557; Berlin.

EIssmann, L. (1953): Mollisoldiapirismus. - Z. angew. Geol., 24: 130 - 138; Berlin.

FreCHEN, J. (1953): Die Herkunft der spätglazialen Bimstuffe in mittel- und süddeutschen Mooren. - Geol. Jb., 67: 209 - 230; Hannover.

JANKOVSKÁ, V. (1980): Paläogeobotanische Rekonstruktion der Vegetationsentwicklung im Becken Tr̃eboňská pánev während des Spätglazials und Holozäns. - Vegetace ČSSR A, 11; Praha.

KNIPPING, M. (1989): Zur spät- und postglazialen Vegetationsgeschichte des Oberpfälzer Waldes. - Dissertationes Botanicae, 140; Berlin-Stuttgart. 
LOŽEK, V. (1964): Quartärmollusken der Tschechoslowakei. - Rozpravy ÚÚG, 17, 374 S.; Praha.

LOŽEK, V. (1965): Das Problem der Lößbildung und der Lößmollusken - Eiszeitalter u. Gegenwart, 16: 61 - 75; Öhringen.

ManiA, D. (1967): Der ehemalige Ascherslebener See in spät- und postglazialer Zeit. - Hercynia, 4: 199 - 260; Leipzig.

- (1968): Ein mittelpaläolithisches Artefakt aus dem Geiseltal. - Ausgrabungen u. Funde, 13: 17 - 19; Berlin.

- (1972): Zur spät- und nacheiszeitlichen Landschaftsgeschichte des mittleren Saalegebietes. - Hall. Jb. mitteldeutsche Erdgesch., 11 (1969): 7 - 36; Leipzig.

- (1973): Paläoökologie, Faunenentwicklung und Stratigraphie des Eiszeitalters im mittleren Elbe-Saalegebiet auf Grund von Molluskengesellschaften. - Geologie, Beiheft 78/79: 175 S.; Berlin

- \& Stechemesser, H. (1969): Eine weichselspätglaziale Molluskensukzession aus dem mitteleuropäischen Periglazialgebiet südlich der Elbe und ihre Bedeutung für die Landschaftsgeschichte. - Petermanns Geogr. Mitt., 113: 1 - 15; Gotha.

- \& STEChemesser, H. (1970): Jungpleistozäne Klimazyklen im Harzvorland. - Petermanns Geogr. Mitt., Ergänzungsheft 274: 39 - 55; Gotha.

- \& TOEPFER, V. (1971): Zur jungquartären Landschaftsgeschichte und mesolithischen Besiedlung des Geiseltals. - Jschr. mitteldt. Vorgesch., 55: 11 - 34; Halle (Saale).
MÜLLER, H. (1953): Zur spät- und nacheiszeitlichen Vegetationsgeschichte des mitteldeutschen Trockengebietes. - Nova Acta Leopoldina, N. F. 16, Nr. 110: 1 - 67; LeipZig.

RuchHolz, K. (1977): Zur Genese gravitativer Schicht- und Sedimentkörperdeformation in Vereisungsgebieten. Wiss. Z. Ernst-Moritz-Arndt-Universität 26, Math.-NatR. 1/2: $49-57$; Greifswald.

- (1979): "Frostbeulen" der mecklenburgischen Landschaft. - Wiss. u. Fortschr., 29: 270 - 274; Berlin.

RYBinickova, E. (1974): Die Entwicklung der Vegetation und Flora im südlichen Teil der Böhmisch-Mährischen Höhe während des Spätglazials und Holozäns. Vegetace ČSSR A, 7; Praha.

Thomae, M. (1986): Zur Genese pleistozäner Lagerungsstörungen im oberen Deckgebirge des Geiseltales. Techn. Kurzinform. (Betriebssektion Kammer u. Technik, Braunkohlenwerk Geiseltal), 41: 20 - 26; Halle (Saale).

Thomae, M. (1990): Geologischer Bau und Lagerungsverhältnisse des Quartärprofils von Neumark-Nord. - Veröff. Landesmus., Vorgesch. Halle, 43: 131 - 143; Berlin.

UsINGER, H. (1985): Pollenstratigraphische, vegetationsund klimageschichtliche Gliederung des "Bölling -Alleröd-Komplexes" in Schleswig-Holstein und ihre Bedeutung für die Spätglazialstratigraphie in benachbarten Gebieten. - Flora, 1/2: 1 - 43; Jena.

Manuskript eingegangen am 13. 3. 1992 